Aus der Abteilung Klinische Neurophysiologie

(Prof. Dr. med. W. Paulus)

im Zentrum Neurologische Medizin

der Medizinischen Fakultät der Universität Göttingen

\title{
Akute Auswirkungen transkranieller Gleichstromstimulation auf Parameter kortikaler Erregbarkeit
}

\author{
INAUGURAL-DISSERTATION
}

zur Erlangung des Doktorgrades

der Medizinischen Fakultät

der Georg-August-Universität zu Göttingen

vorgelegt von

\section{Cornelia-Carmen Sturhan}

geb. Klein

aus

Osterode am Harz

Göttingen 2011 
Dekan:

I. Berichterstatter:

II. Berichterstatter/in:

III. Berichterstatter/in:

IV. Berichterstatter/in:

Tag der mündlichen Prüfung:
Prof. Dr. med. C. Frömmel

Prof. Dr. med. M. Nitsche

Prof. Dr. med. Hülsmann

Prof. Dr. med. Brockmann

Prof. Dr. med. Oppermann

24. Juli 2012 


\section{Inhaltsverzeichnis}

$1 \quad$ Einleitung 1

2 Material und Methoden

2.1 Probanden 7

2.2 Erzeugung von motorkortikalen Erregbarkeitsveränderungen durch tDCS 8

2.3 Erfassung der Exzitabilität des motorischen Kortex mit TMS

2.4 Index für Exzitabilität des kortikospinalen Kerngebietes der Muskelrepräsentation: motorische Ruheschwelle

2.5 Index der Exzitabilität des kortikospinalen Kerngebietes der Muskelrepräsentation : aktive motorische Schwelle.

2.6 Index der kortikospinalen Aktivität größerer Neuronenpopulationen: Input-OutputKurve

2.7 Index der intrakortikalen Exzitabilität: Intrakortikale Inhibition und Fazilitierung..... 12

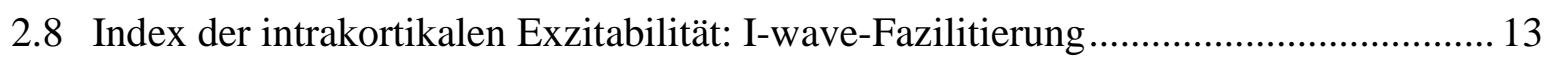

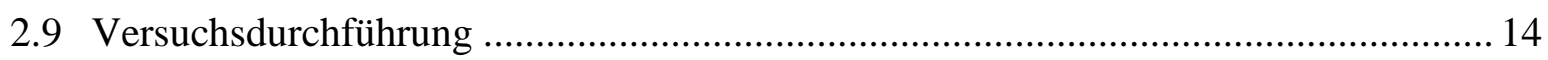

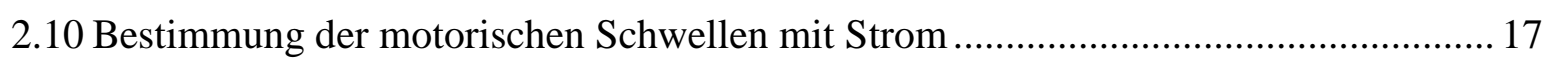

2.11 Erfassung der Rekrutierung, intrakortikalen Fazilitierung/ Inhibition und I-wave-

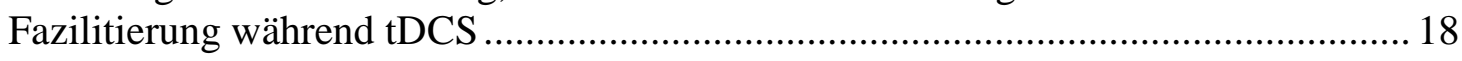

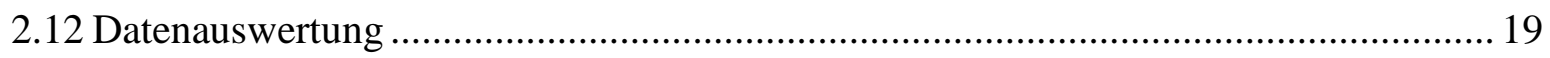

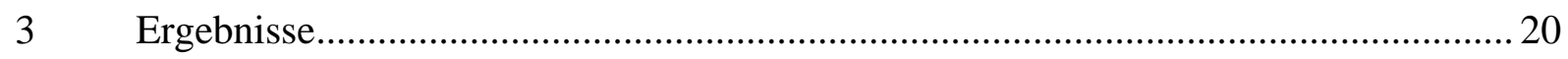

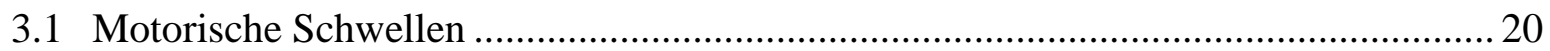

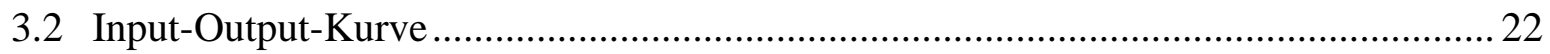

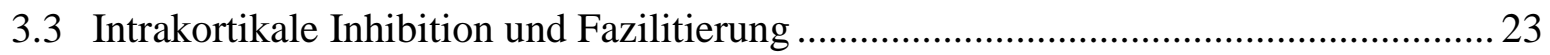

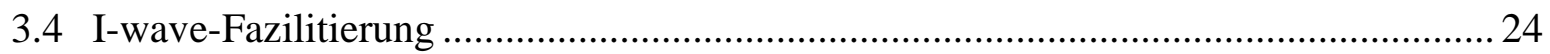

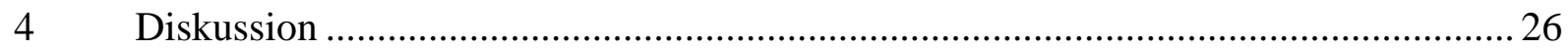

4.1 Polaritätsspezifische Änderungen der kortikospinalen Erregbarkeit hinsichtlich der IO-Kurvenprotokolle. Jedoch keine Effekte von tDCS auf motorische Schwellen ...... 26

4.2 Geringe Modulation intrakortikaler Inhibition und Fazilitation durch tDCS.............. 28

4.3 Fehlende Wirkung von tDCS auf die I-wave-Aktivität............................................. 29

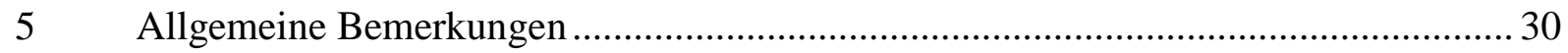

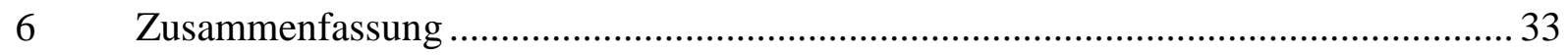

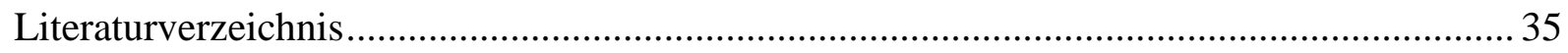




\section{Abbildungsverzeichnis}

Abbildung 1: TMS-induzierter Strom und Magnetfeld im zeitlichen Verlauf......................... 10

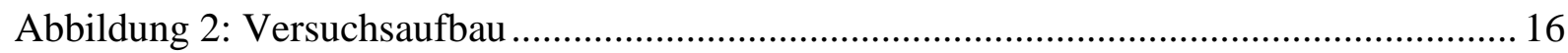

Abbildung 3: Darstellung der Ruheschwelle und der aktiven motorischen Schwelle. ............ 21

Abbildung 4: tDCS verändert die Amplitude der Input/Output-Kurve polaritätsspezifisch.... 22

Abbildung 5: Intrakortikale Inhibition und Fazilitierung werden durch tDCS moduliert. ...... 23

Abbildung 6: I-wave-Fazilitierung wird durch tDCS nicht moduliert .................................. 24

\section{Tabellenverzeichnis}

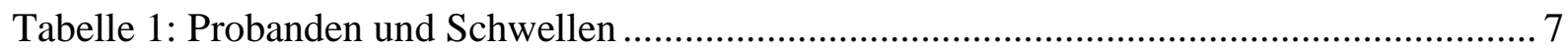

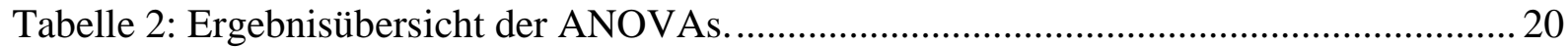




\section{Abkürzungsverzeichnis}

Abb. Abbildung

ADM Musculus abductor digiti minimi

Ag $\quad$ Argentum (Silber)

Ag-Cl Silberchlorid

AMT Aktive Motorische Schwelle (engl. active motor threshold)

AMTA Aktive Motorische Schwelle anodal (engl. active motor threshold anodal)

AMTC Aktive Motorische Schwelle kathodal (engl. active motor threshold cathodal)

AMTN Aktive Motorische Schwelle ohne Strom (engl. active motor threshold non DC)

ANOVA Varianzanalyse (engl. analysis of variance)

$\mathrm{Ca} \quad$ Kalzium

Cl Chlor

$\mathrm{cm} \quad$ Zentimeter

Dig. $\quad$ Finger (lat. digitus)

etc. et cetera

EMG Elektromyogramm

engl. englisch

ev. evangelisch

e.V. eingetragener Verein

f weiblich (engl. female)

fMRI funktionelle Kernspintomographie

(engl. functional magnetic resonance imaging)

FSJ freiwilliges soziales Jahr 
GABA $\quad \gamma$-Aminobuttersäure (engl. $\gamma$ - amino-butyric-acid)

h Stunde (engl. hour)

$\mathrm{Hz} \quad$ Hertz

ISI Interstimulusintervall

K Kalium

lat. lateinisch

LTD Langzeitdepression (engl. long-term depression)

LTP Langzeitpotenzierung (engl. long-term potentiation)

M männlich (engl. male)

$\mathrm{mA} \quad$ Milliampere

$\min \quad$ Minuten

mm Millimeter

MSAP Muskelsummenaktionspotential

ms Millisekunden

$\mathrm{mV} \quad$ Millivolt

$\mathrm{m} \Omega \quad$ Milliohm

n.a. nicht anwendbar

NMDA N-Methyl-D-Aspartat

PAS gepaarte assoziative Stimulation (engl. paired associative stimulation)

PET Positronen-Emissions-Tomographie

RMT Ruhemotorschwelle (engl. resting motor threshold)

RMTA Ruhemotorschwelle anodal (engl. resting motor threshold anodal)

RMTC Ruhemotorschwelle kathodal (engl. resting motor threshold cathodal) 
RMTN Ruhemotorschwelle ohne Strom (engl. resting motor threshold non-DC)

rTMS repetitive transkranielle Magnetstimulation

S

Sekunden

S.

Seite

$\mathrm{Sd}$

Standardabweichung

s.o. $\quad$ siehe oben

SPECT Single-Photon-Emissions-Computertomographie

tDCS transkranielle Gleichstromstimulation

(engl. transcranial direct current stimulation)

TES transkranielle elektrische Stimulation

TMS transkranielle Magnetstimulation

TP Testpuls

u.a. unter anderem

UK United Kingdom

vgl. vergleiche

z.B. zum Beispiel

ZNS zentrales Nervensystem

z.Zt. $\quad$ zur Zeit 


\section{Einleitung}

Neuroplastizität wird als Fähigkeit des Gehirns beschrieben, seine eigene Struktur und Organisation veränderten biologischen Grundlagen (Läsionen) und Anforderungen (veränderte Umweltbedingungen) anzupassen. Selbst primitive Nervensysteme besitzen die Fähigkeit, aufgrund von Erfahrungen sinnvolle Änderungen der Reaktionsweise des Systems zu generieren: sie lernen. Das Wissen um die physiologischen Grundlagen neuronaler Plastizität ist von besonderem Interesse.

Die Abhängigkeit neuronaler Signalübertragung von elektrischer Aktivität als mögliche Grundlage von Neuroplastizität war bereits im 19. Jahrhundert bekannt. Die Induzierbarkeit motorischer Akte durch elektrische Stimulation des zentralen Nervensystems (ZNS) wurde am offenen Kortex tierexperimentell belegt (Ferrier 1875, Fritsch und Hitzig 1870)

Vorwiegend zur genaueren Charakterisierung kortikaler Areale wurden die Versuche an Primaten (Leyton und Sherrington 1917) und später auch an neurochirurgischen Patienten systematisch ausgeweitet. Eine Stimulation des motorischen Kortex durch die intakte Schädelkalotte war allerdings zunächst nicht möglich.

Mit Einführung der TES gelang Merton und Morton 1980 erstmals die elektrische Stimulation eines humanen Motorkortex durch die intakte Schädeldecke hindurch. Die verwendete transkranielle elektrische Stimulation (TES), bei der mittels durch die Schädeldecke applizierter elektrischer Hochvoltreize der motorische Kortex gereizt und in Folge an den Extremitätenmuskeln ein Summenaktionspotential abgeleitet werden konnte, stimulierte jedoch nicht ausschließlich die Neurone des motorischen Kortex, sondern auch kutane Nozizeptoren, so dass diese Methode aufgrund ihrer Schmerzhaftigkeit problematisch war. Eine schmerzfreie und nicht invasive Methode zur Stimulation des menschlichen Motorkortex fand sich 1985 durch Barker et al.. in der Entwicklung der transkraniellen Magnetstimulation (TMS). Durch sie können, durch eine flach auf den Kopf aufgelegte Magnetspule und durch diese applizierte starke Magnetimpulse, intrakortikal elektrische Ströme induziert werden, die ihrerseits zu einer Erregung neuronaler Strukturen führen. Vorzugsweise wird dieses Verfahren im Bereich des primären Motorkortex verwendet, da sich das Ausmaß der Reizung als Muskelsummenaktionspotential (MSAP) am Zielmuskel aufzeichnen lässt.

Durch die Entwicklung weiterer nicht invasiver Verfahren, wie repetitive Transkranielle 
Magnetstimulation (rTMS), Positronen-Emissions-Tomographie (PET), funktionelle Kernspintomographie (fMRI), gepaarte assoziative Stimulation (PAS) und EinzelphotonenEmissions-Tomographie (SPECT), hat die Erforschung bzw. der Nachweis von zerebraler Erregbarkeit und Aktivität als Grundlage der Neuroplastizität innerhalb der Neurowissenschaften einen großen Fortschritt erfahren. Oben genannte Methoden sind dabei $u$. a. in der Lage, Plastizität zu erzeugen und Funktionen neuronaler Verbände bildgebend darzustellen. Verfahren wie TMS erlauben nicht nur die akute Erfassung von zerebraler Erregbarkeit, sondern auch den Nachweis überdauernder Veränderungen.

Sowohl tierexperimentell (Rioult-Pedotti et al. 2000) als auch im Humanversuch (Grafton et al. 1995, 1998, Pascual-Leone et al. 1994) wurde die Abhängigkeit von Lernvorgängen von Veränderungen kortikaler Erregbarkeit herausgestellt. Von besonderem Interesse sind hier insbesondere längerfristige Veränderungen der Effizienz synaptischer Übertragungen. Zwei Formen überdauernder Erregbarkeitsveränderungen gelten hier als neuronale Grundlage des Lernens: Langzeitpotenzierung (LTP) und Langzeitdepression (LTD). Dabei kann noch stunden- bis tagelang nach Erzeugung von hoch- bzw. niedrigfrequenten Serien von Aktionspotentialen am synaptischen Eingang eine potenzierte bzw. reduzierte synaptische Übertragung gemessen werden (Bliss und Lomo 1973, Buonomano 1999, Castro-Almancos und Calcagnotto 1999, Eysel et al. 1998, Hess und Donoghue 1994, Krug et al. 1984)

Mit Entwicklung der repetitiven TMS (rTMS) (Charlton et al. 2003, Siebner et al. 2004) wurde ein Verfahren entwickelt, um eben diese lang anhaltenden Erregbarkeitsveränderungen im humanen Motorkortex (und auch weiteren Kortizes) zu erzeugen und auch hier LTP oder LTD zu induzieren. Ähnlich ist das Verfahren der gepaarten assoziativen Stimulation (PAS). Auch hier wird durch eine repetitive, phasische, assoziative Stimulation eine Aktivierung von Neuronen erzielt. Konventionelle rTMS-Protokolle können jedoch aufgrund der engen Sicherheitsbestimmungen nur begrenzt verwendet werden (Wassermann 1998). Eine Sonderform der rTMS stellt die Theta-Burst-Stimulation dar, deren Stimulationsprotokolle eher den tierexperimentellen Protokollen zur Erzeugung von LTP und LTD entsprechen (Huang et al. 2005).

Ein weiteres Verfahren, um kortikale Aktivität zu verändern und somit Neuroplastizität zu erzeugen, besteht in der zerebralen Stimulation mit schwachem Gleichstrom (Bindmann et al. 1964; Creutzfeld et al. 1962; Gartside 1968a,1968b; Purpura und McMurtry 1965). Die Gleichstromstimulation diente ursprünglich im Tierversuch der Modulation von kortikaler 
Exzitabilität und Spontanaktivität. Im Vergleich zu bereits erwähnten Verfahren besteht das Funktionsprinzip hier nicht in einer repetitiven, phasischen Stimulation, bei der mutmaßlich synchrone und assoziative Aktivierung der Neurone zu den genannten Effekten führt, sondern in einer unterschwelligen Ruhemembranpotentialauslenkung kortikaler Neurone mittels tonischer transkranieller Applikation eines Gleichstroms über die intakte Kopfhaut. Während die Wirkung der PAS als synapsenspezifisch gilt, zeigen sich tDCS und rTMS bezüglich der Qualität der erzielten Effekte vergleichbar. Beide Verfahren führen zu globalen Erregbarkeitsveränderungen neuronaler Systeme.

Die Verschiebung des neuronalen Ruhemembranpotentials mittels tDCS führt zu einer unterschwelligen De - oder Hyperpolarisation neuronaler Membranen (Purpura und McMurtry 1965; Creutzfeld et al. 1962). Anodale Polarisierung führt dabei zu einem depolarisierenden Effekt an Neuronenmembranen und kathodale Polarisierung zu einer Hyperpolarisierung. Daraus ergibt sich eine Erregbarkeitssteigerung der genannten Neurone bei anodaler Stimulation und eine verminderte Erregbarkeit bei kathodaler Polarisation. (Bindmann et al. 1964; Creutzfeld et al. 1962; Gartside 1968b; Purpura und McMurtry 1965; Terzuolo und Bullock 1956).

Die erreichten Effekte erweisen sich allerdings als instabil und überdauern bei einer kurzen Stimulationsdauer (unter 5min) die Polarisierung nicht. Bei längerer Stimulationsdauer können jedoch messbare Effekte bis zu 5h nach Polarisation nachgewiesen werden (Bindmann et al. 1964; Gartside 1968a, 1968b).

Die zu Beginn verwendete Methode der intrakraniellen Gleichtromstimulation wurde Ende des 20. Jahrhunderts durch das Verfahren der transkraniellen Stimulation mit schwachem Gleichstrom (tDCS) ergänzt. Diese Methode bot die Vorteile eines nicht-invasiven Verfahrens bei gleichzeitig ausreichender Stromstärke am Wirkort (ca. 50\% des transkraniell applizierten Gleichstroms) (Rush und Driscoll 1968).

Die Ergebnisse sind denen der tierexperimentellen Untersuchungen vergleichbar: anodale Stimulation erhöht und kathodale Stimulation vermindert die Exzitabilität (Nitsche und Paulus 2000). Mutmaßlich sind die Effekt intrakortikal lokalisiert, da die Exzitabilität des kortikospinalen Traktes unverändert bleibt (Nitsche und Paulus 2000; 2001; Nitsche et al. 2003a). Wie in kürzlich durchgeführten, pharmakologischen Studien beschrieben, hängen die durch kurz andauernde Stromstimulation hervorgerufenen Effekte, welche keine Nacheffekte vorweisen, von der Aktivität der Natrium- und Calciumkanäle ab. Veränderungen an NMDA (n- 
Methyl-d-Aspartat)- oder GABA ( $\gamma$ - Amino-Buttersäure)-Rezeptoren spielen hier keine Rolle (Nitsche et al. 2004a). Somit sind die Effekte während Stimulation möglicherweise allein durch polaritätsspezifische Änderungen des Ruhemembranpotentials ausgelöst. Nacheffekte nach lang anhaltender Stimulation hingegen sollten, wie auch durch Tierversuche nahegelegt, durch Veränderung synaptischer Stärke hervorgerufen werden (Gartside 1968b). Neuere Studien weisen hier auf den NMDA-Rezeptor als zentralen Wirkort hin (Liebetanz et al. 2002, Nitsche et al. 2003b, 2004a, b).

Insgesamt zeigen die Nacheffekte bezüglich der NMDA-Rezeptorabhängigkeit Ähnlichkeit mit tierexperimentellen, Neuroplastizität erzeugenden Paradigmata der LTP und LTD.

Dennoch ist, abgesehen von diesen pharmakologischen Studien, wenig über die durch tDCS modulierten, neuronalen Systeme bekannt. Im Hinblick auf die einfache, schmerzlose und nicht invasive Anwendung sowie die vielversprechenden Ergebnisse im Rahmen der neuronalen Plastizität ist die Erforschung der genauen neurophysiologischen Zusammenhänge der kortikalen Erregbarkeitsänderungen durch tDCS von großer Bedeutung. Neben den bereits beschriebenen Auswirkungen von tDCS auf den motorischen Kortex sind z.B. funktionelle Auswirkungen auf den visuellen Kortex, untersucht mittels visuell evozierter Potentiale (Antal et al. 2004), die Beeinflussung motorischen Lernens, bestätigt durch ein Serial-ReactionTime-Task (Nitsche et al. 2003c), und die Beeinflussung klinischer Symptome bei Patienten, z.B. Leistungsverbesserung motorischer Fähigkeiten bei postapoplektischen Paresen (Fregni et al. 2005, Hummel et al. 2005), bekannt.

Diese Arbeit befasst sich mit der Frage nach dem Ursprung der Erregbarkeitsänderung während der Stimulation mit schwachem Gleichstrom, der keine Nacheffekte erzeugt.

$\mathrm{Zu}$ diesem Zweck wurden TMS-Protokolle verwendet, welche bekanntermaßen spezifische kortikale, neuronale Systeme erfassen und Veränderungen während tDCS aufzeigen können.

Aktive motorische Schwelle, motorische Ruheschwelle und Input-Output-Kurven repräsentieren Messprotokolle der kortikospinalen Exzitabilität (Abruzzese und Trompetto 2002; Chen 2000). Die Motorische Schwelle ist definiert als die minimale TMS-Intensität, aus der noch ein MSAP resultiert. Dabei wird für die motorische Ruheschwelle am relaxierten Muskel und für die aktive motorische Schwelle am moderat kontrahierten Muskel gemessen

Unter Medikation mit Natriumkanalblockern kommt es zu einer Erhöhung der Schwellen (Ziemann et al.1996), keine Veränderung zeigt sich jedoch bei Gammaaminobuttersäure 
(GABA)- oder Glutamatrezeptoren beeinflussenden Medikamenten (Ziemann et al. 1996, 1998a). Somit reflektiert die motorische Schwelle neuronale Membranaktivität. Definitionsgemäß befinden sich die TMS-Intensitäten innerhalb des MT-Protokolls auf Schwellenniveau, daher wird durch sie die Erregbarkeit einer zentralen Kernregion innerhalb der muskelrepräsentierenden, kortikalen Region betrachtet.

Im Vergleich mit den motorischen Schwellen gibt die I-O-Kurve Auskunft über größere Neuronenpopulationen. Mit steigender TMS-Intensität kommt es zu einer Rekrutierung von immer größeren Neuronenverbänden, dies wird anhand des Kurvenverlaufes deutlich. Auch bei der IO-Kurve findet sich eine Beeinflussung durch Natriumkanalblocker, ihre Steigung wird durch die Gabe von Natrium- und Calciumkanalblockern verringert und somit eine Abhängigkeit von der Membranexzitabilität bestätigt.

Zur Untersuchung intrakortikaler Inhibition und Fazilitation sind TMSDoppelstimulationsprotokolle geeignet (Kujirai et al. 1993). Dazu folgt einem unterschwelligen, nicht kontraktionsauslösenden Reiz ein überschwelliger Teststimulus. Die durch den Teststimulus hervorgerufene MSAP-Amplitude weist in Abhängigkeit vom zeitlichen Abstand zum präkonditionierenden unterschwelligen Stimulus eine Inhibition oder Fazilitation intrakortikalen Ursprungs auf und reflektiert die Erregbarkeit inhibitorischer und exzitatorischer Interneurone (Kujirai et al. 1993). Da Inhibition durch GABAerge und antiglutamaterge Substanzen verstärkt und Fazilitation durch selbige supprimiert wird, beide jedoch nicht durch die Applikation von Ionen-Kanal-Blockern beeinflusst werden (Chen 1997; Liepert et al. 1997; Ziemann et al. 1996, 1998a), reflektieren Inhibition und Fazilitation hauptsächlich die Aktivität des glutamatergen und des GABAergen Systems im Motorkortex.

Als I-Waves werden indirekte, kortikospinale Wellen des Motorkortex bezeichnet, die der ersten (direkten) Welle nach Stimulation des Motorkortex folgen. Sie werden durch intrakortikale neuronale Kreise kontrolliert (Ziemann und Rothwell 2000). Ihre Untersuchung erfolgt durch ein weiteres Doppelstimulationsprotokoll (Ziemann et al.1998a). Dazu folgt einem überschwelligen Teststimulus ein zweiter unterschwelliger Reiz in definiertem zeitlichem Abstand. Die daraus resultierende Vergrößerung der abgeleiteten MSAP-Amplitude ist spezifisch für die verwendeten Interstimulusintervalle (ISI) und reflektiert mutmaßlich kortikale Interaktionen zwischen den Neuronenkreisen, die dem Ursprung indirekter kortiko-spinaler Wellen dienen. Da es durch Gabe von GABAergen Medikamenten zu einer Reduktion der IWave-Fazilitation kommt, nicht jedoch durch eine Applikation von Ionen-Kanal-Blockern 
(Ziemann et al. 1998c), zeigt sich durch I-waves die Aktivität des GABAergen Systems im Motorkortex.

Ziel der vorliegenden Studie ist die Exploration der unmittelbaren Effekte der tDCS auf intrakortikale und kortikospinale Neuronenpopulationen mittels der oben genannten TMSTechniken. Hierdurch soll eine genauere Charakterisierung des Wirkmechanismus der tDCS ermöglicht werden. 


\section{Material und Methoden}

\subsection{Probanden}

Die Versuche wurden, abhängig vom Untersuchungsprotokoll, an 12 bis 20 gesunden Probanden (männlich / weiblich : 65\% / 35\%) mit einem Durchschnittsalter von 26 Jahren durchgeführt. (Tab. 1) An der Untersuchung für Ruheschwelle, Aktive Motorische Schwelle und Doppelstimulation nahmen, mit Ausnahme von 2 Personen im Versuch der Aktiven Motorischen Schwelle, die gleiche Gruppe von Probanden teil. Jeweils andere Probandengruppen wurden für die Untersuchungen der Rekrutierung und der I-Wave-Aktivität eingesetzt.

Tabelle 1: Probanden und Schwellen

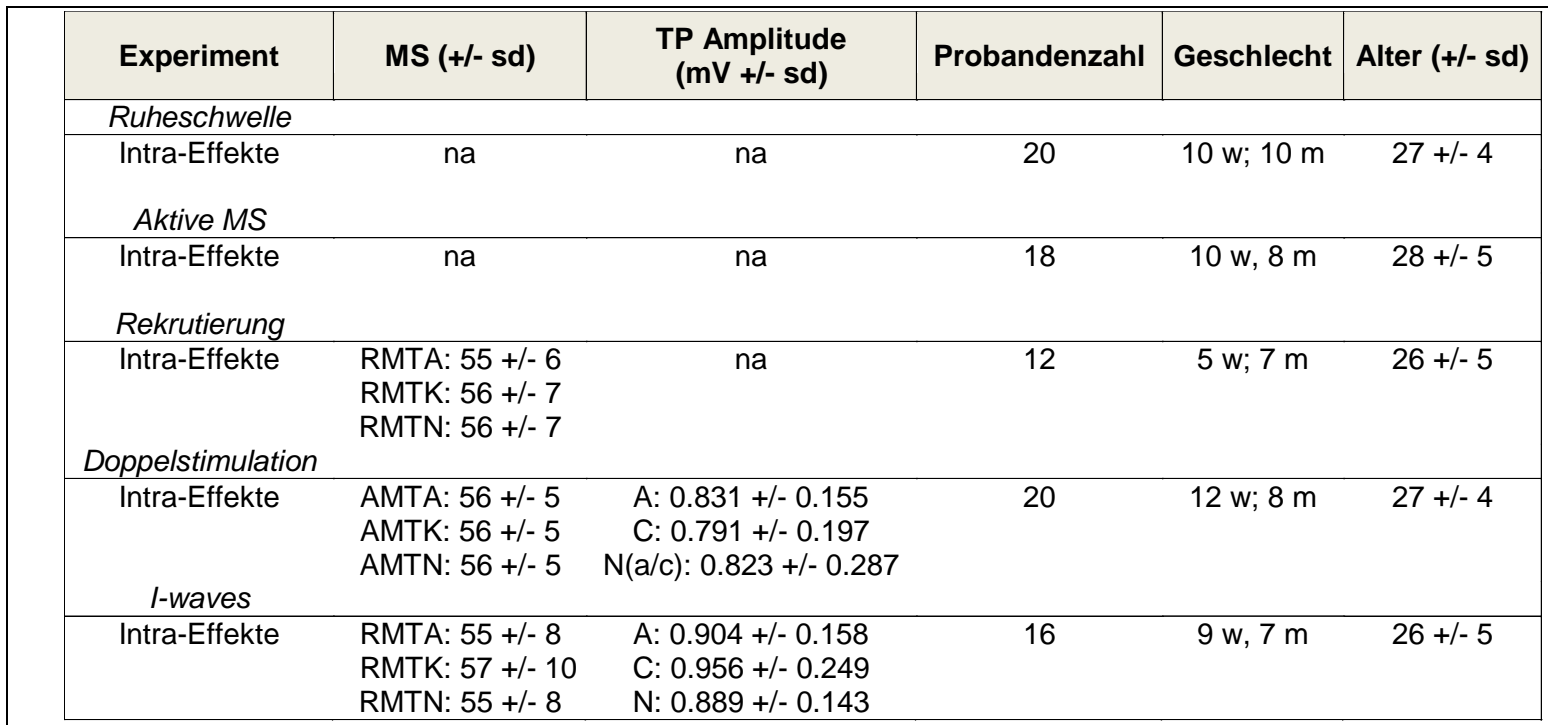

Die vorliegenden Daten sind als Mittelwerte + /- Standardabweichung dargestellt. Das Experiment bezieht sich auf die für jede tDCS-Bedingung verwendeten Versuchsprotokolle (intra-tDCS). Für jeden Versuch wurden 12 20 Probanden getestet; Alter und Geschlecht zeigten sich zwischen allen Versuchen vergleichbar. Prozentuale Angabe der maximalen Stimulatorleistung. Die Mittel der Motorische Schwellen und Einzeltestpulsamplituden ohne Gleichstromstimulation unterschieden sich nicht signifikant zwischen den einzelnen Bedingungen (Student'scher t-Test, p>0.05; w, weiblich; m, männlich; n.a, nicht anwendbar).

Voraussetzungen für die Teilnahme waren körperliche und geistige Gesundheit, erfasst durch Anamnese und eine orientierende körperliche Untersuchung. Drogenmissbrauch und die dauerhafte oder aktuelle Einnahme von zentralnervös wirkenden Substanzen führten zum Ausschluss von der Studie. 
Alle Personen wurden vor Versuchsbeginn schriftlich und mündlich genau über den Aufbau und die Versuchsdurchführung informiert, ebenso über eventuelle Nebenwirkungen von TMS und tDCS (Müdigkeit, Kopfschmerz) oder mögliche Sensationen während der Versuchsdurchführung (Lichtblitze, Kribbelparästhesien) .

Zum Ausschluss von der Studie führten die in der Probandenaufklärung aufgeführten Kontraindikationen und Ausschlusskriterien.

Nach einem zweiten, vom Versuchsleiter persönlich durchgeführten Aufklärungsgespräch, gaben die Probanden ihr schriftliches Einverständnis.

Die Teilnahme an den Experimenten wurde durch ein Probandengeld vergütet.

Alle Experimente wurden durch die Ethikkommission der Universität Göttingen begutachtet und genehmigt (Antragsnummer: 15/4/99).

\subsection{Erzeugung von motorkortikalen Erregbarkeitsveränderungen durch tDCS}

Die Applikation von schwachem Gleichstrom über die intakte Kopfhaut erfolgte über ein Paar gut angefeuchteter Schwammelektroden mit einer Fläche von $35 \mathrm{~cm}^{2}$. Die Stromabgabe erfolgte über einen speziell entwickelten, batteriebetriebenen Stromstimulator (Schneider Elektronic, Gleichen, Germany). Mit dem Gerät kann eine maximale Stromstärke von 2 mA (Milliampère) erreicht werden.

Die Motor-Kortex-Elektrode wurde auf das den rechten Musculus abductor digiti minimi (ADM) repräsentierende Areal des motorischen Kortex platziert. Der optimale Stimulationspunkt wurde vorab mittels TMS festgelegt. Die zweite Elektrode wurde über der kontralateralen Orbita befestigt. Die Stromapplikation erfolgte für jeweils 4 Sekunden (s) (Intra-tDCS) mit einer Stromstärke von $1 \mathrm{~mA}$.

Die Kontrolle eines stetigen und gleichmäßigen Stromflusses wurde durch ein zwischen- geschaltetes Voltmeter gewährleistet.

Während der Stromapplikation kam es bei einigen Versuchsteilnehmern zu leichten Hautirritationen (Jucken, leichtes Stechen) im Bereich der Elektroden. 
Nahezu jeder Proband konnte unmittelbar nach Ein und-/oder Ausstellen des Stromstimulators einen Lichtblitz wahrnehmen.

Um Interferenzen zwischen den einzelnen tDCS-Bedingungen, insbesondere bei dem I-waveProtokoll, bei dem es insgesamt zu einer relativ häufigen Stromapplikation kam, auszuschlieBen, wurde zwischen den einzelnen Stimulationsprotokollen eine Pause von einer Woche eingehalten.

\subsection{Erfassung der Exzitabilität des motorischen Kortex mit TMS}

Die transkranielle Hirnstimulation mit Magnetfeldimpulsen (transkranielle Magnetstimulation; TMS) hat sich aufgrund der nebenwirkungsarmen und schmerzlosen Durchführung für die Reizung des motorischen Kortex bewährt. Durch einen Strompuls, welcher durch eine Reizspule fließt, wird ein Magnetimpuls generiert, der seinerseits intrakortikal Reizströme induziert (Hess und Lundin, 1988, siehe Abb.1). Dieser Mechanismus entspricht dem Prinzip der elektromagnetischen Induktion. Der Reizstrom erregt die Pyramidenbahnzellen im Vergleich zur elektrischen Kortexreizung vermutlich transsynaptisch. Reizt man also den motorischen Kortex mit einem Magnetstimulator und leitet die Muskelantwort von z.B. einem Handmuskel ab, erhält man die Summe aus zentraler und peripherer Leitungszeit plus der Verzögerung durch zwei zwischengeschaltete Synapsen. 
Abbildung 1: TMS-induzierter Strom und Magnetfeld im zeitlichen Verlauf

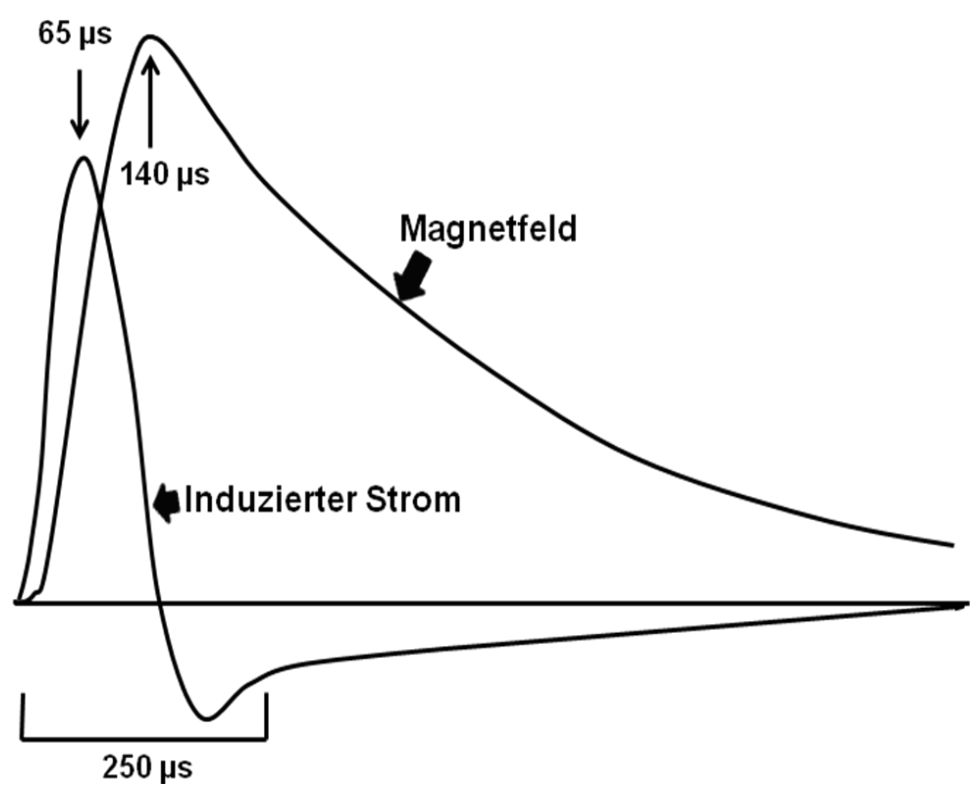

Typischer zeitlicher Verlauf des Magnetfeldes und des induzierten Stromes eines Magnetstimulators (Reizspule mit $12 \mathrm{~m} \Omega$ Impedanz und $35 \mu \mathrm{H}$ Selbstinduktion. Der induzierte Strom folgt der ersten Ableitung des Magnetfeldes. (Hess und Lundin, 1988, S. 210)

\subsection{Index für Exzitabilität des kortikospinalen Kerngebietes der Muskel- repräsentation: \\ motorische Ruheschwelle}

Als motorische Ruheschwelle ist die minimale TMS-Stimulusintensität definiert, bei der wenigstens drei von sechs der erzeugten MSAP (Muskelsummenaktionspotentiale) am entspannten Muskel eine Peak-zu-Peak-Amplitude von $50 \mu \mathrm{V}$ oder mehr aufweisen.

Die Bestimmung ging aus von einer zuvor bestimmten Baseline, bei der das durchschnittliche MSAP eine Größe von $2 \mathrm{mV}$ haben sollte.

Davon ausgehend wurde die Intensität dann in Schritten von 1-2\% so lange reduziert, bis die maximale Intensität, bei der das oben genannte Kriterium nicht mehr erfüllt wurde, erreicht war. $\mathrm{Zu}$ der so bestimmten Stimulatorintensität wurde $1 \%$ hinzugefügt und diese Intensität als motorische Ruheschwelle definiert.

Essentiell zur optimalen Bestimmung der motorischen Ruheschwelle ist eine maximale Muskelentspannung der Versuchsperson.

Eine willkürliche oder unwillkürliche Mus- kelkontraktion führt zu einer Vergrößerung 
der MSAP-Amplituden und somit zu einer falsch niedrigen Schwellenbestimmung.

Durch einen zwischengeschalteten Oszillographen war es den Probanden und dem Versuchsleiter möglich, die Muskelspannung und Entspannung visuell und akustisch zu kontrollieren.

\subsection{Index der Exzitabilität des kortikospinalen Kerngebietes der Muskel- repräsentation : aktive motorische Schwelle}

Die aktive Schwelle (AMT, engl. active motor threshold) ist definiert als die minimale Stimulatorintensität, bei der das ausgelöste MSAP in drei von sechs aufeinander folgenden Stimuli in Größe und Konfiguration von der Hintergrundaktivität des aktivierten Zielmuskels eindeutig zu unterscheiden ist.

Durch die willkürliche Kontraktion oder Vorspannung des Muskels kommt es zu einer gesteigerten Erregbarkeit der kortikospinalen Bahnen, so dass zur Depolarisation ein Reiz niedrigerer Intensität ausreicht und die AMT somit einen niedrigeren Wert annimmt als die RMT (Hess und Lundin, 1988).

In diesem Falle wurde die Kontraktion des Zielmuskels durch willkürliche Abduktion des Dig. V erreicht (ca. 15\% der maximalen Kontraktionskraft).

Über ein zwischengeschaltetes Oszilloskop konnte die Muskelaktivität über optische und akustische Rückkopplung kontrolliert werden.

Ausgehend von einem überschwelligen Teststimulus wurde die Stimulatorintensität in Schritten von 1-2 \% verringert bis zur höchsten Intensität, bei der das oben genannte Kriterium nicht mehr erfüllt wurde. Zu der so bestimmten Stimulatorintensität wurde dann $1 \%$ hinzugefügt und diese Intensität dann als aktive motorische Schwelle definiert. 


\subsection{Index der kortikospinalen Aktivität größerer Neuronenpopulationen: Input-Output-Kurve}

Die Messung der I-O-Kurven gibt einen Einblick in die Erregbarkeit größerer kortikospinaler Neuronengruppen.

Dazu wurden TMS-Intensitäten von 100\%, 110\%, 130\% und 150\% der zuvor bestimmten Ruheschwelle verwendet. Die Applikation erfolgte in randomisierten Blocks mit jeweils 15 Stimuli pro Block.

Die Steilheit der resultierenden Kurven reflektiert die Rekrutierung größerer neuronaler kortikospinaler Neuronenpopulationen durch steigende TMS-Intensitäten.

\subsection{Index der intrakortikalen Exzitabilität: Intrakortikale Inhibition und Fazilitierung}

Durch Verwendung eines Doppelstimulationsprotokolls wird die Erfassung der kortikalen Erregbarkeit ermöglicht. Dazu wird dem individuell festgelegten Teststimulus ein konditionierender Reiz (CS) in einem definierten zeitlichen Abstand vorausgesetzt. Die Abstände variieren nach Versuchsaufbau und werden als Interstimulusintervalle (ISI) bezeichnet

Als ISI wurden 2, 3, 5, 10 und 15 Millisekunden (ms) verwendet, wobei die ersten drei ISI inhibitorische und die letzten zwei ISI fazilitatorische Effekte hervorrufen (Kujirai et al. 1993)

Als Teststimulus (TS) ist die Stimulatorintensität definiert, mit der am entspannten Muskel ein MSAP mit einer Peak-zu Peak -Amplitude von $1 \mathrm{mV}$ hervorgebracht werden kann. Unter Versuchsbedingungen wurde ein einzelner, isoliert applizierter und nicht konditionierter Reiz als Kontrollreiz bezeichnet.

Als konditionierender Stimulus wurde eine Stimulatorintensität von 70\% der zuvor bestimmten aktiven motorischen Schwelle definiert. Diese geringe Stimulationsintensität wurde gewählt, um leicht provozierbare Decken- oder Bodeneffekte zu vermeiden, welche einen möglichen Einfluss der tDCS maskiert hätten. Ein zu starker inhibitorischer oder fazilitatorischer Einfluss des konditionierenden Stimulus bewirkt möglicherweise eine Verkleinerung oder gar 
das Verschwinden eventueller tDCS- Effekte.

Durch die Konditionierung eines Testreizes werden die nachfolgenden MSAP im Vergleich zum nicht konditionierten Kontrollreiz in ihrer Amplitude verändert. Die Veränderungen resultieren aus einer durch die ISI hervorgerufene Mehr - oder Mindererregbarkeit der beteiligten Neurone. Dabei bezeichnet man ein MSAP als fazilitiert, wenn es eine größere Amplitude aufweist als das Kontroll-MSAP, als inhibiert, wenn es in der Größe verringert ist.

Innerhalb des Messdurchgangs wurde in allen Protokollen die Stimulationsintensität gegebenenfalls adjustiert, um mögliche Effekte der tDCS auf die Testpulsamplitude in Form von Erregungsänderungen zu kompensieren.

Alle Konditionen werden pseudorandomisiert und wiederholt gemessen, um Effekte durch spontan auftretende Schwankungen zu minimieren (Kiers et al. 1993).

Die Wiederholungen jeder Bedingung werden gemittelt und durch den Mittelwert der ebenfalls pseudorandomisierten Kontrollreizwiederholungen dividiert.

Die einzelnen Blöcke wurden 15mal wiederholt, wobei die Reihenfolge der einzelnen Stimuluspaare innerhalb der Blöcke pseudorandomisiert appliziert wurden.

\subsection{Index der intrakortikalen Exzitabilität: I-wave-Fazilitierung}

Die Ermittlung der I-wave-Fazilitierung erfolgte mittels eines Doppelstimulationsprotokolls, bei welchem der TMS-Teststimulus dem konditionierenden Stimulus vorangestellt wurde. (Ziemann et al. 1998b)

Die ISI lagen zwischen 0.5 und $5.1 \mathrm{~ms}$ mit einem Abstand von 0,2 ms.

Die Stimuluspaare wurden zusammengefasst in fünf pseudorandomisierten Blöcken und mit einem Zeitintervall von 4 s zwischen den einzelnen Stimulationspaaren appliziert und aufgezeichnet. 
Die Messung der einzelnen Blöcke wurde 15-mal wiederholt.

Jeder Block beinhaltete fünf Doppelstimulationspaare und einen Einzeltestpuls.

Für den Einzelstimulus wurde die Stimulatorintensität definiert, bei der ein Baseline-MSAP von ca. $1 \mathrm{mV}$ abgeleitet werden konnte.

Auch hier wurde während des aktuellen Messdurchgangs in allen Protokollen die Stimulationsintensität gegebenenfalls adjustiert, um mögliche Effekte von tDCS auf die Testpulsamplitude in Form von Erregungsänderungen zu kompensieren.

Für den konditionierenden Stimulus wurde eine Stimulatorintensität von $70 \%$ der zuvor bestimmten Ruhemotorschwelle festgelegt, um eventuelle Decken- und Bodeneffekte durch zu hohe Intensitäten zu vermeiden (Ziemann et al.1998b).

Eine deutliche Vergrößerung der EMG-Antwort ist bei ISI 1.1-1.5, 2.3-2.9 und 4.1-4.4 zu erwarten (Hess und Donoghue 1994).

\subsection{Versuchsdurchführung}

Im Folgenden wird der allgemeine Versuchsaufbau und - ablauf zur Ermittlung der kortikalen Exzitabilität mittels TMS sowie die praktische Durchführung zum Aufsuchen des optimalen Stimulationspunktes und die Erfassung der Erregbarkeitsänderungen während der Stromapplikation dargelegt.

Für alle Experimente wurden die Probanden in einem eigens dafür umgebauten Zahnarztstuhl mit einer vielfach verstellbaren Kopfstütze, Armlehnen und Rückenlehne platziert, um eine möglichst bequeme und haltungsstabile Sitzposition zu gewährleisten.

Zur Oberflächenelektromyographie wurden $\mathrm{Ag}-\mathrm{AgCl}-$ Elektroden (Durchmesser 9mm) verwendet.

Abgeleitet wurde am ADM, der als intrinsischer Handmuskel mit großflächiger und oberflächennaher kortikaler Repräsentation für Magnetstimulationsstudien sehr geeignet ist.

Eine differente Elektrode wurde dabei direkt über dem Muskelbauch, die indifferente Elektrode über dem distalen Sehnenansatz platziert. 
Befestigt wurden die Elektroden mit handelsüblichem, hautfreundlichem Pflaster. Zur Verbesserung der Ableitung und Gewährleistung eines konstanten Kontaktes, auch bei langer Versuchsdauer, wurden die Elektroden vor endgültiger Befestigung mit einem Elektrodenkontaktgel versehen.

Eine großflächige Erdungselektrode wurde am Unterarm angebracht (s. Abb. 2).

Das von den beiden Oberflächenelektroden aufgenommene Signal wurde vorverstärkt, mit einer Zeitkonstante von $5 \mathrm{~s}$ und einem Tiefpassfilter von $2,5 \mathrm{kHz}$ gefiltert und mit $5000 \mathrm{~Hz}$ digitalisiert (Kamen und Caldwell 1996) und nun mit einem IBM-kompatiblen Pentium 133 $\mathrm{MHz}$ Computer aufgezeichnet. Als Aufzeichnungsprogramm wurde NEUROScan verwendet (Version 3.2, NEUROScan Inc.; Sterling, VA, USA).

Die Magnetstimulation erfolgte durch zwei Magstim 200 Stimulatoren, die über jeweils 2 Booster-Module und mit einem BiStim-Modul verschaltet waren, so dass die von beiden Geräten generierten Stimuli zusammen auf eine Magnetspule geleitet wurden (alle Geräte: The Magstim Company Ltd., Whitland, Dyfed, UK).

Um eine möglichst selektive und fokale Stimulation des kortikalen Repräsentationsfeldes des Zielmuskels zu erreichen, wurde eine Magnetspule in Form einer Acht mit einem Windungsdurchmesser von $70 \mathrm{~mm}$ verwendet. In der Schnittstelle der Windungen kommt es zur maximalen Stimulationsintensität.

Die bei diesem Versuchsaufbau maximal erreichbare Stimulationsintensität betrug ca. 2,2 Tesla. Nachfolgend werden die verwendeten Intensitäten als Prozent dieser Maximalintensität angegeben.

Die Stimulatoren wurden über einen IBM-kompatiblen PC getriggert.

Die Software wurde von Dr. rer. nat. Baudewig und Dr .med. F. Tergau (Abteilung Klinische Neurophysiologie der Universität Göttingen) programmiert. Dieser Versuchsaufbau gewährleistete eine definierte Ausgangsleistung der Stimulatoren innerhalb von 100ms. Die Triggerung konnte mit einer Genauigkeit von $0,1 \mathrm{~ms}$ erfolgen.

Der optimale Stimulationspunkt repräsentiert das Areal über dem motorischen Kortex, auf dem mit einem Testreiz das größtmögliche MSAP des abgeleiteten Muskels (ADM) provoziert werden kann. Dazu wurde die Magnetspule im $45^{\circ}$-Winkel zur Sagittalebene des Kopfes aufgelegt. Da in allen Fällen vom rechten ADM abgeleitet wurde, musste sich das Repräsen- 
tationsfeld auf der linken Hemisphäre befinden, etwa auf einer Sagittalebene zwischen Scheitel und linker Orbita im mittleren bis hinteren Drittel. Die Spule musste der Krümmung des Schädels entsprechend so austariert werden, dass sie zu allen Seiten den gleichen Abstand vom Kopf hatte (Mills et al.1992).

In dieser Haltung wurde die Magnetspule dann in 0,5-1-cm-Abständen von frontal nach dorsal und medio-lateral bewegt. In einem Intervall von $4 \mathrm{~s}$ wurden leicht überschwellige Testreize appliziert, die in der Lage waren ein MSAP des ADM auszulösen. Im Vergleich wurde der Punkt als Optimum bestimmt, auf dem bei gleich bleibender Intensität das größte MSAP hervorgerufen und zudem wiederholt reproduziert werden konnte. Hier wurde dann mit einem wasserfesten Stift eine Markierung auf der Kopfhaut angebracht.

Die optimale Lokalisation wurde vor jeder Messung individuell und neu bestimmt.

Folgten mehrere Versuchsdurchläufe an einem Tag, wurde der auf der Kopfhaut markierte optimale Stimulationspunkt wieder verwendet.

\section{Abbildung 2: Versuchsaufbau}

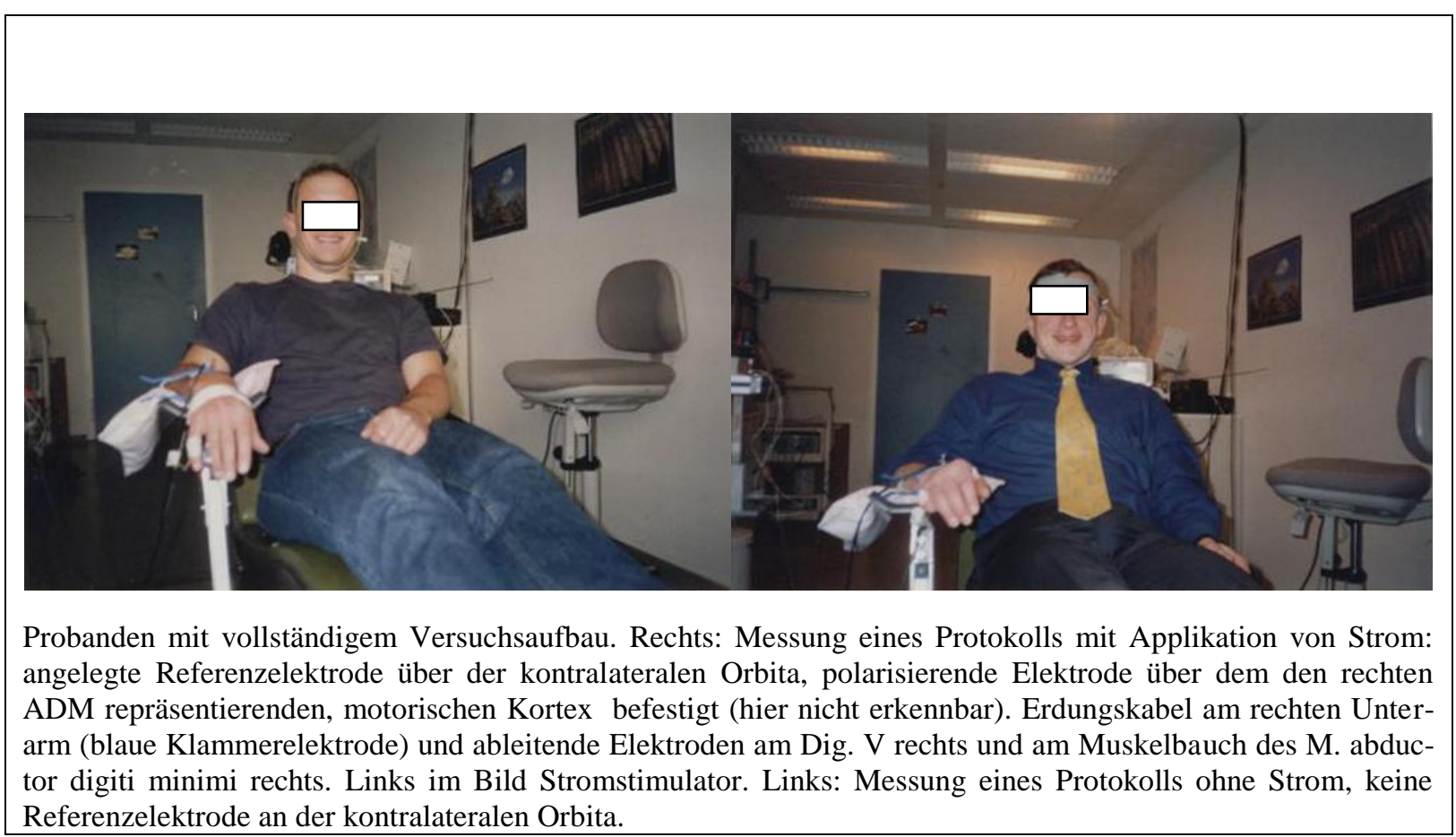




\subsection{Bestimmung der motorischen Schwellen mit Strom}

Bei allen tDCS- Versuchen wurden sämtliche motorische Schwellen während des Stromflusses bestimmt. Hier gelten die gleichen Ausgangsbedingungen wie bei Bestimmung ohne Strom. Additiv wurde während der Schwellenbestimmung mittels TMS ein schwacher Gleichstrom von 1mA appliziert. Begonnen wurde mit der Bestimmung der Baseline, einem gering überschwelligen Teststimulus. Um das Schwellenniveau zu erreichen, wurde die Stimulationsintensität im Verlauf jeweils um 1-2 \% verringert bis zu einer Stimulatorintensität, bei der das ausgelöste MSAP in drei von sechs aufeinander folgenden Stimuli eine Peak-zuPeak-Amplitude von $50 \mu \mathrm{V}$ betrug. Die ermittelte Stimulationsintensität wurde am Stimulator abgelesen und analog zur Ruheschwellenbestimmung ohne Strom 1\% addiert und dann als motorische Ruheschwelle mit Strom festgelegt.

Die Bestimmung der aktiven Schwelle erfolgte ebenfalls unter Applikation von tDCS, wobei die Kriterien zur Auffindung der gesuchten Schwelle denen der Bestimmung ohne Stromstimulation identisch sind. Ausgehend von einem überschwelligen Teststimulus wurde die Stimulatorintensiät in Schritten von 1-2\% so lange reduziert, bis bei aktiviertem Muskel (ADM) das ausgelöste MSAP in Größe und Konfiguration gerade nicht mehr von der registrierten Hintergrundaktivität zu unterscheiden ist. Zu der ermittelten Stimulatorintensität wurde 1\% addiert und dieser Wert als aktive motorische Schwelle festgelegt.

Diese Schwellenbestimmungen mit Strom erfolgten jeweils für kathodale und anodale tDCS, wobei zwischen beiden Bestimmungen eine Pause von mind. 10 Minuten lag, um eine Verfälschung der Ergebnisse durch verbliebene Effekte des Stromes auf die kortikospinale Erregbarkeit zu vermeiden. 


\subsection{Erfassung der Rekrutierung, intrakortikalen Fazilitierung/ Inhibition und I-wave-Fazilitierung während tDCS}

Um während tDCS auftretende Erregbarkeitsänderungen zu bestimmen, wurden 200ms vor Ende einer $4 \mathrm{~s}$ dauernden tDCS durch TMS generierte MSAP ausgelöst und aufgezeichnet. Dazu wurde die wie oben beschriebene Protokolle (I-O-Kurve, Inhibition/ Fazilitierung, Iwaves) verwendet.

Die Experimente wurden dabei in je drei Sitzungen durchgeführt, bei denen entweder anodale oder kathodale tDCS, sowie eine Kontrollmessung des jeweiligen Protokolls ohne Stromreizung angewendet wurden, jedoch unter sonst identischen Versuchsbedingungen (gleiche Lagerung, Anlegen der Schwammelektroden etc.). Vor Beginn des Doppelstimulationsprotokolls wurde individuell die TMS- Intensität bestimmt, die ein MSAP von $1 \mathrm{mV}$ Größe, ein so genanntes Baseline-MSAP erzeugten.

Die Applikation der Protokolle erfolgte inter-individuell randomisiert.

Um störende Effekte durch auftretende Müdigkeit des Probanden zu verhindern, wurde die maximale Dauer eines Versuchsdurchlaufes auf zwei Stunden limitiert.

Mit Ausnahme des I-wave-Protokolls, welches aufgrund der großen Stimulusanzahl für die verschiedenen Strom- und Nichtstrombedingungen an verschiedenen Tagen durchgeführt wurde, wurden die anderen Doppelstimulationsprotokolle mit Pausen an einem Versuchstag durchgeführt. 


\subsection{Datenauswertung}

Zum Vergleich der Motorischen Schwellen wurden die inter - individuellen Mittelwerte der TMS- Intensitäten für aktive Schwelle und Ruheschwelle für tDCS und Nicht-tDCSBedingungen (intra-Effekte und anodale und kathodale tDCS) gesondert berechnet. Für beide Stimulationsbedingungen wurden die Ergebnisse ohne tDCS unter Verwendung von Student'schen t-Tests (gepaart, zweiseitig, Signifikanzgrenze 0,05) verglichen mit denen der Versuchsreihe während tDCS.

Im Hinblick auf die anderen Protokolle wurden bezüglich der verschiedenen Konditionen (anodal, kathodal, ohne Strom) zunächst die Mittelwerte der intra- individuellen MSAPAmplituden für jede TMS-Stimulation-Bedingung (TMS- Intensität im Hinblick auf die I-OKurve, ISI im Hinblick auf die intrakortikale Inhibition/Fazilitierung und I-waveFazilitierung) einzeln für anodale, kathodale oder non-tDCS-Bedingungen berechnet. Für die Doppelstimulationsprotokolle wurden die vorhandenen Ergebnisse an denen der respektiven Einzelpulskondition standardisiert und dann die interindividuellen Mittelwerte jeder Kondition berechnet. Für alle Protokolle wurden Varianzanalysen berechnet (ANOVAs, unabhängige Variablen ISI und DC (anodal/kathodal/ohne Strom), abhängige Variable: MSAP - Amplitude; für die I-O- Protokoll- Kurven: unabhängige Variablen: Intensität und DC, abhängige Variable: MSAP-Amplitude).

Unterschiede zwischen den einzelnen DC-Bedingungen einerseits und der non-tDCSBedingung andererseits wurden dann durch post-hoc-Mittelwertvergleiche bestimmt. Ebenfalls wurde durch Einzelvergleiche überprüft, ob sich die Kontrollreize voneinander unterschieden Hier kamen post hoc Fisher's LSD Tests (kritisches $p<0,05)$ zur Anwendung. 


\section{Ergebnisse}

\subsection{Motorische Schwellen}

Wie die entsprechenden t-Tests $(\mathrm{P}>0,05)$ zeigen (siehe Tab 1), differieren die motorischen Schwellen der tDCS-Bedingung und die motorischen Schwellen der Vergleichsbedingung ohne Stromapplikation tDCS (intra-tDCS Effekte) nicht bezüglich der aktiven motorischen Schwelle und der motorischen Ruheschwelle.

Tabelle 2: Ergebnisübersicht der ANOVAs.

\begin{tabular}{|c|c|c|c|c|}
\hline Versuch & & Freiheitsgrade & F-value & $\mathbf{p}$ \\
\hline \multicolumn{5}{|l|}{ I/O Kurve } \\
\hline \multirow[t]{3}{*}{ Intra DC } & $\mathrm{DC}$ & 2 & 17.763 & $<0.001^{*}$ \\
\hline & $\begin{array}{l}\text { TMS } \\
\text { Intensität }\end{array}$ & 3 & 52.101 & $<0.001^{*}$ \\
\hline & $\begin{array}{l}\text { DC x TMS } \\
\text { Intensität }\end{array}$ & 6 & 5.053 & $<0.001^{*}$ \\
\hline \multicolumn{5}{|c|}{ Doppelstimulation } \\
\hline \multirow[t]{3}{*}{ Intra-tDCS } & DC & 2 & 6.947 & $0.003 *$ \\
\hline & ISI & 4 & 23.408 & $<0.001^{*}$ \\
\hline & DC x ISI & 8 & 1.975 & 0.053 \\
\hline \multicolumn{5}{|l|}{ I-waves } \\
\hline \multirow[t]{3}{*}{ Intra-tDCS } & $\mathrm{DC}$ & 2 & 0.023 & 0.977 \\
\hline & ISI & 23 & 4.846 & $<0.001^{*}$ \\
\hline & DC $x$ ISI & 46 & 1.095 & 0.312 \\
\hline
\end{tabular}

Die ANOVAs wurden für die Intra-tDCS-Bedingung mit Blick auf die Protokolle für I-O-Kurve, Intrakortikale Inhibition und Fazilitierung und I-waves berechnet. Die Intra-Effekte beziehen sich auf ein 4sec. tDCSProtokoll, welches keine Nacheffekte hervorruft. Wie die Ergebnisse der ANOVAs zeigen, moduliert IntratDCS I-O-Kurve und intrakortikale Inhibition und Fazilitierung, während verändernde Effekte von tDCS auf Iwaves nicht hervorgerufen werden konnten.

Es ergeben sich für die Intra-tDCS-Messungen eine Ruhemotorschwelle von 54.9 +- $5.9 \%$ der maximalen Stimulatorleistung für die nicht-tDCS-Bedingung (anodale Kontrolle), 54.7+$5.8 \%$ für die anodale tDCS Bedingung, 55.0+- 5.6\% für die nicht-tDCS-Bedingung (kathodale Kontrolle) und 54.9 +- 5.9\% für die kathodale tDCS-Bedingung (siehe Tab 1). 
Das Mittel der aktiven Schwelle für intra-tDCS liegt für die Kontrollbedingung ohne Strom (anodale Kontrolle) bei 44.7 +- 8.2\% der maximalen Stimulatorleistung, für die anodale Bedingung wies das Mittel einen Wert von 44.5 +- $8.6 \%$ auf, $44.5+-8.3 \%$ für die Kontrollbedingung ohne tDCS (kathodale Kontrolle) und 44.6 +- $7.9 \%$ für die kathodale Bedingung (siehe Abb. 3)

Abbildung 3: Darstellung der Ruheschwelle und der aktiven motorischen Schwelle.

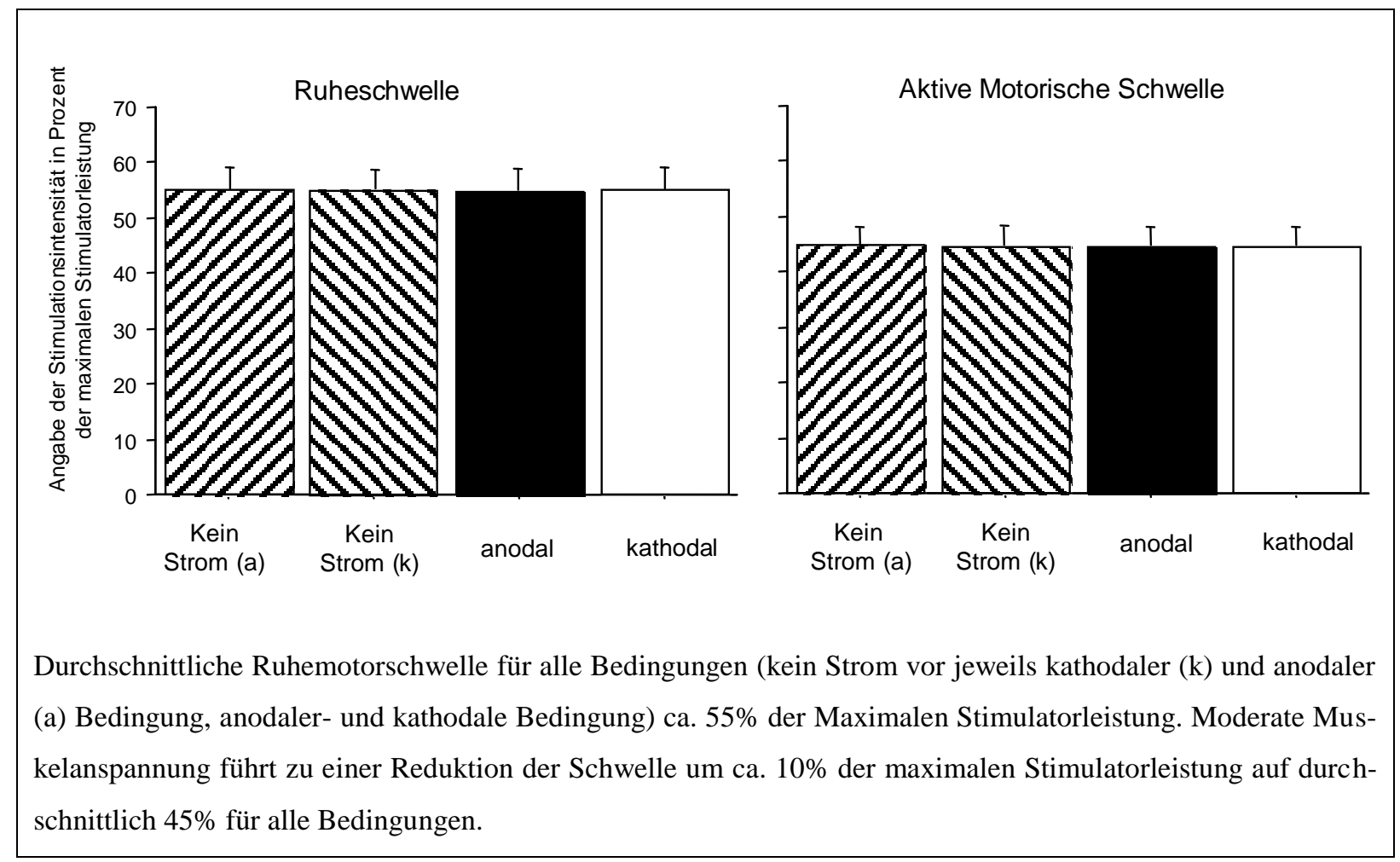




\subsection{Input-Output-Kurve}

Die Varianzanalyse (s. Tab.2) zeigt signifikante Ergebnisse für die Haupteffekte TMS- Intensität, tDCS und für die Interaktion beider Parameter (4 s tDCS, MSAP ausgelöst während der Stromstimulation).

Die graphische Darstellung (Abb. 4) zeigt eine deutliche Zunahme der MSAP-Amplituden unter steigender Stimulatorintensität

Abbildung4: tDCS verändert die Amplitude der Input/Output-Kurve polaritätsspezifisch

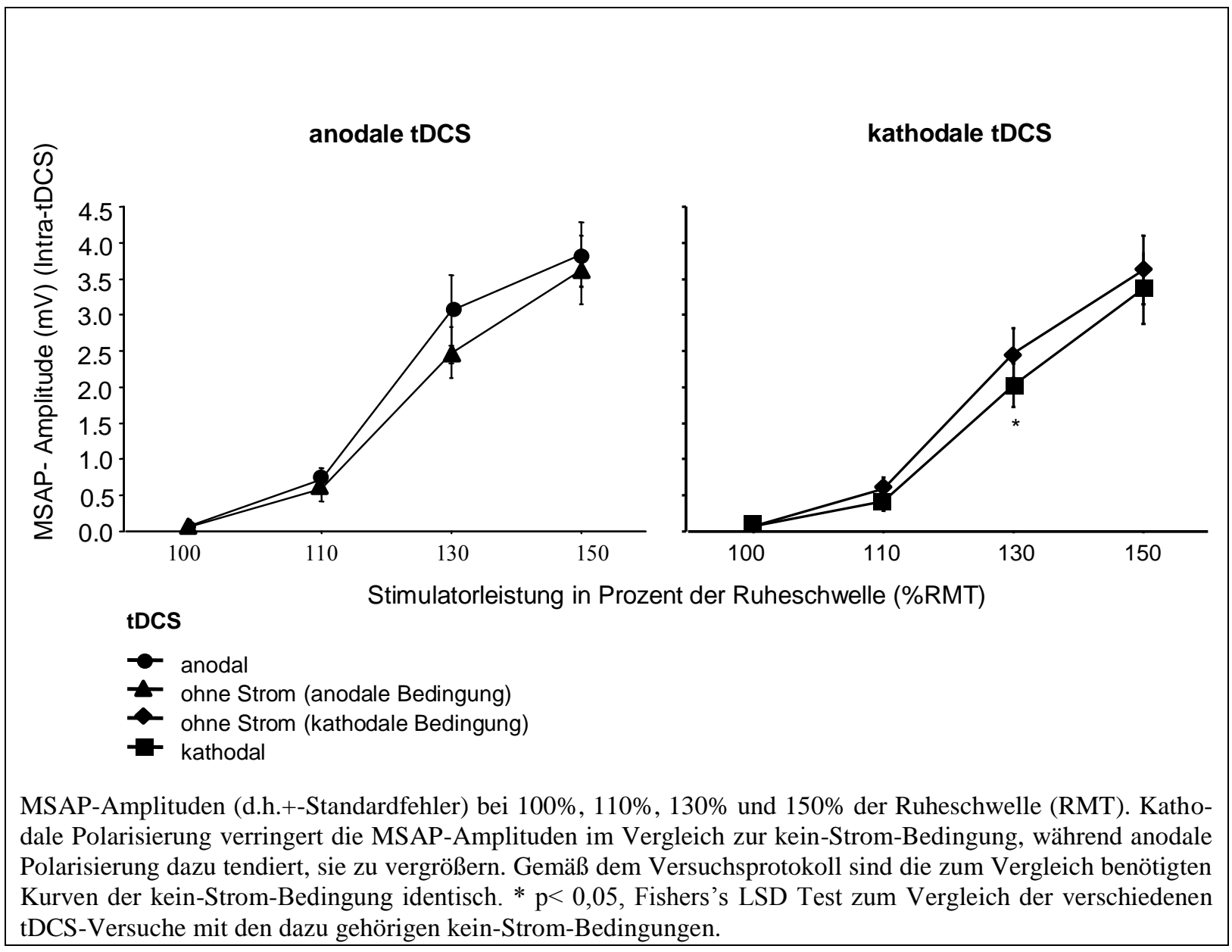

Unter kathodaler tDCS erfolgt eine signifikante Reduktion der in der unter (non-tDCS) Vergleichsbedingungen erzielten MSAP-Vergrößerung für die Intensität 130\% der Ruheschwelle (Fisher's LSD Test, $\mathrm{p}<0.05$ ).

Anodale Stimulation mit tDCS führt hingegen nur zu einer nicht signifikanten Tendenz in 
Richtung MSAP-Vergrößerung (gesteigerter Erregbarkeit, s. Abbildung 4) gegenüber der Kondition ohne tDCS.

\subsection{Intrakortikale Inhibition und Fazilitierung}

Für das Intra-tDCS-Protokoll zeigt die Varianzanalyse (siehe Tab. 2) signifikante Effekte in Bezug auf tDCS und Interstimulusintervalle (ISI).

Die Interaktion beider Faktoren war hingegen nicht signifikant.

Wie die post-hoc-Einzelvergleiche zeigten, wurden unter anodaler Stromstimulation keine signifikanten Veränderungen der kortikalen Inhibition oder Fazilitierung erzielt. Kathodale tDCS reduzierte die Fazilitierung bei einem ISI von 15ms signifikant gegenüber der Kontrollbedingung ohne tDCS (siehe Abb. 5).

Abbildung 5: Intrakortikale Inhibition und Fazilitierung werden durch tDCS moduliert.

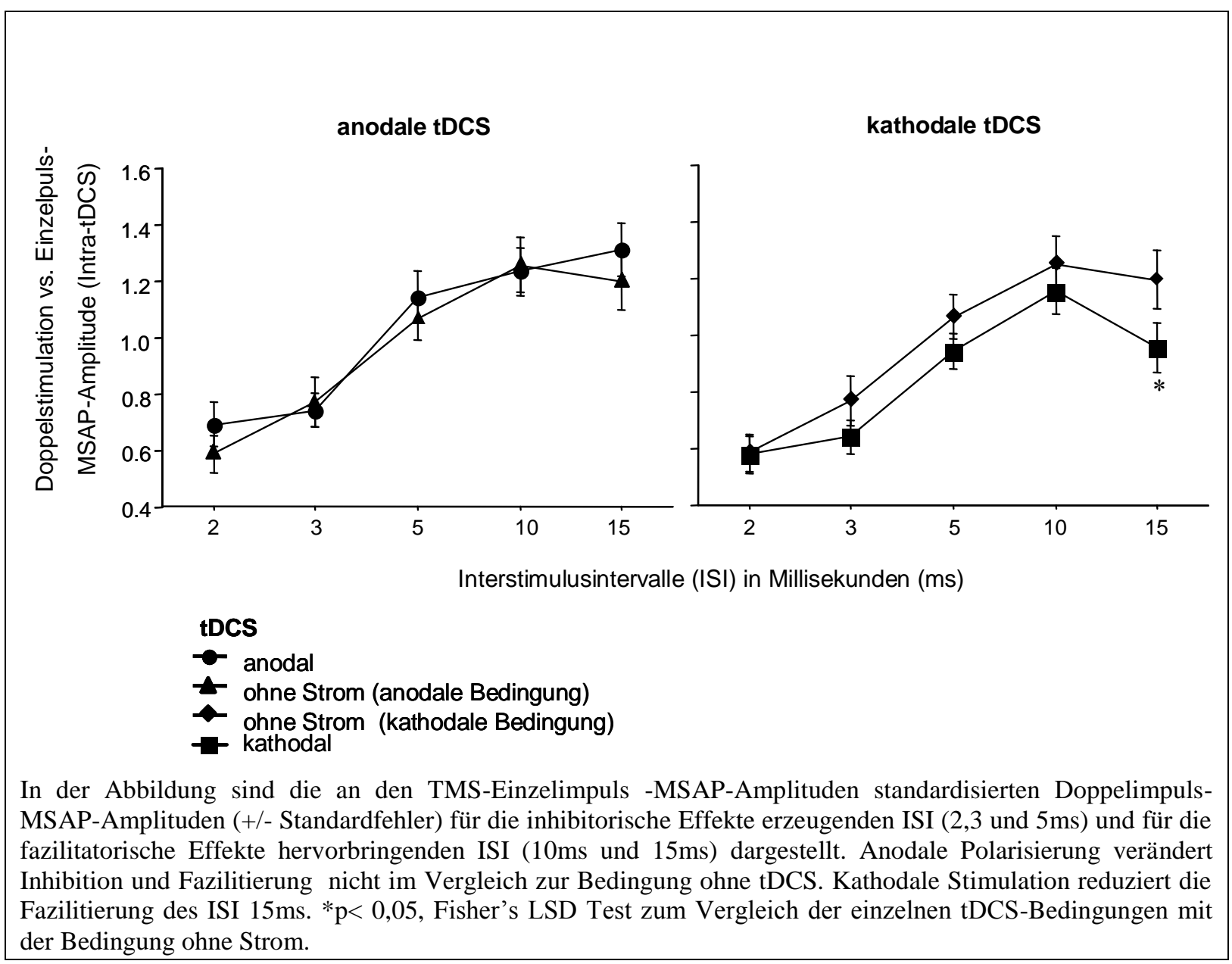




\subsection{I-wave-Fazilitierung}

Die Varianzanalyse ANOVA (s. Tab. 2) zeigt für die intra-tDCS-Bedingung einen signifikanten Haupteffekt für die Interstimulusintervalle (ISI).

Hingegen waren der Haupteffekt von tDCS und die Interaktion zwischen ISI und tDCS, anders als für das verwendete Doppelstimulationsprotokoll (Kujirai et al. 1993), nicht signifikant. Wie in Abb. 6 dargestellt, kam es durch Applikation von tDCS nicht zu einer Veränderung der I-wave-Fazilitierung .

Abbildung 6: I-wave-Fazilitierung wird durch tDCS nicht moduliert

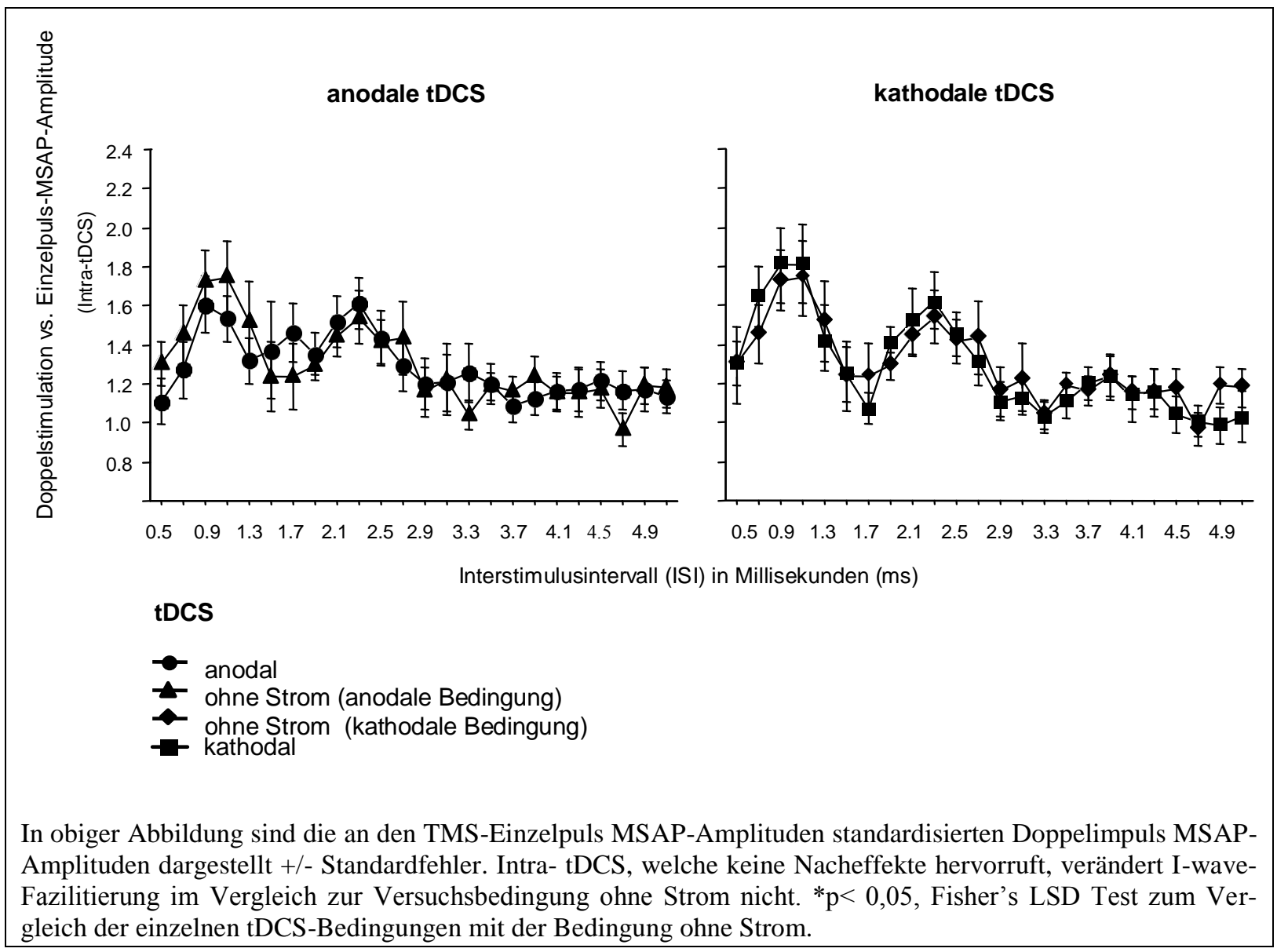


Die Motorischen Schwellen wiesen keine signifikante Differenz unter den verschiedenen tDCS-Bedingungen (z.B. tDCS, keine tDCS, vor tDCS, nach tDCS) in den einzelnen Protokollen auf. Die Einzelpuls-Test-Amplituden waren identisch für alle Protokolle in allen Konditionen (Tab. 1). 


\section{Diskussion}

In dieser Studie wurde mittels TMS der Frage nachgegangen, welche kortikospinalen und intrakortikalen neuronalen Systeme im Rahmen einer Stimulation mit schwachem Gleichstrom, welcher keine Nacheffekte auslöst, moduliert werden.

\subsection{Polaritätsspezifische Änderungen der kortikospinalen Erregbarkeit hinsichtlich der I-O-Kurvenprotokolle. Jedoch keine Effekte von tDCS auf motorische Schwellen}

Die Ergebnisse der beiden Versuchsprotokolle, welche zum Nachweis von Änderungen kortikospinaler Erregbarkeit durchgeführt wurden, zeigen unterschiedliche Ergebnisse.

In Bezug auf die Schwellenuntersuchung zeigen sich keine Veränderungen der aktiven motorischen Schwelle und der motorischen Ruheschwelle während der Applikation von tDCS. Die Ergebnisse zur Bestimmung der I-O-Kurven stimmen dagegen im Wesentlichen mit den bislang bekannten Ergebnissen für Einzel-Puls-TMS-Stimulation überein (Nitsche und Paulus 2000, 2001; Nitsche et al. 2003a). Stimulation mit anodalem Gleichstrom resultierte in einem Trend zur Vergrößerung der MSAP-Amplitude, während es unter kathodaler tDCS zu einer Größenreduktion kam.

Diese Effekte sind damit vereinbar, dass es abhängig von der verwendeten Polarität zu einer Modulation der kortikospinalen Erregbarkeit kommt, wodurch nach einem definierten TMSImpuls eine unterschiedliche Anzahl von Neuronenpopulationen rekrutiert und aktiviert wird.

Anodale tDCS vergrößert die Anzahl rekrutierter Neurone, während kathodale tDCS diese vermindert. Diese Ergebnisse stimmen bezüglich der I-O-Kurve im Wesentlichen mit voraus gegangenen Einzel-Puls-TMS-Untersuchungen überein (Nitsche und Paulus 2000).

Dass die Effekte der tDCS auf die I-O-Kurve unter kathodaler tDCS deutlicher sind als unter anodaler Stromstimulation, stimmt mit tierexperimentellen Befunden überein, die zeigen, dass es einfacher ist, eine Reduktion der kortikalen Erregbarkeit zu erzeugen, als umgekehrt die Steigerung der kortikalen Erregbarkeit (Malenka und Bear 2004). Möglicherweise erzeugt also kathodale tDCS stabilere Effekte als anodale, wie es auch für die Nacheffekte der tDCS 
gesichert ist (Nitsche und Paulus 2001, Nitsche et al. 2003a). Eine weitere mögliche Erklärung für die Asymmetrie der Effekte ist ein Deckeneffekt der Membranerregbarkeit, welcher bezüglich der Intra-Effekte keine weitere Erregbarkeitssteigerung durch anodale tDCS bei hohen TMS-Intensitäten zulässt. Gestützt wird diese Vermutung durch die Tatsache, dass anodale tDCS dazu tendiert, kortikale Erregbarkeit nur unter Verwendung moderater TMSIntensitäten zu steigern (Nitsche et al. 2005). Deckeneffekte wären allerdings nicht bei niedrigen und moderaten TMS-Intensitäten zu erwarten, so dass diese als Erklärung für das Fehlen signifikanter anodaler Effekte nicht ausreichen.

Im Hinblick auf den Mechanismus der tDCS stimmen die Ergebnisse bezüglich der I-OKurve mit früheren Tierversuchen überein, in denen polaritätsspezifische de- oder hyperpolarisierende Effekte auf neuronale Membranen während schwacher Gleichstromstimulation beschrieben wurden (Purpura und McMurtry 1965). Auch weisen neuere pharmakologische Studien auf ähnliche membranpolarisierende Mechanismen der tDCS beim Menschen hin. So kommt es unter Gabe von Ionenkanalblockern zu einer Reduktion der durch tDCS induzierten Erregbarkeitssteigerung, da die I-O-Kurve wesentlich von der Aktivität von Ionenkanälen abhängt (Nische et al. 2002, Nitsche et al. 2003c). Die Blockade von glutamatergen Rezeptoren hingegen weist keinen Effekt auf die I-O-Kurve auf, da diese nicht von der NMDARezeptoraktivität abhängig sind (Nitsche et al. 2003c)

Es bedarf einer Erklärung, warum die I-O-Kurven eine Veränderung durch tDCS erfahren, nicht jedoch die motorischen Schwellen, obwohl beide Protokolle die kortikospinale Erregbarkeit erfassen und von der Membranpolarisierung abhängen. Im Vergleich zur Bestimmung der motorischen Schwellen werden bei der Erhebung der I-O-Kurve deutlich größere Neuronenpopulationen rekrutiert und aktiviert (Chen 2000). So kann vermutet werden, dass die Sensitivität des I-O-Kurven-Protokolls zur Erfassung von Änderungen der kortikospinalen Erregbarkeit vergleichbar höher ist, als das Protokoll zur Bestimmung der motorischen Schwellen. 


\subsection{Geringe Modulation intrakortikaler Inhibition und Fazilitation durch tDCS}

Die Ergebnisse der durchgeführten Intra-tDCS-Protokolle zeigen lediglich eine geringe Modulation von Fazilitation und Inhibition durch Applikation von tDCS.

Anodale Polarisation erzeugte keinerlei signifikante Modifikation der intrakortikalen Erregbarkeit. Kathodale Stimulation hingegen bewirkte eine Verminderung der Fazilitierung für ein ISI (15ms).

Das Fehlen eines wirksamen Effektes bezüglich der anodalen Polarisation lässt sich mit der NMDA Rezeptor-Abhängigkeit der Doppelstimulationseffekte erklären (Ziemann et al.1996). Wie eine frühere Studie zeigte, verändert eine kurze tDCS das Ruhemembranpotential, hat aber keine Auswirkungen auf NMDA-Rezeptoren (Nitsche et al. 2003b)

Kathodale Stimulation hingegen bewirkt eine signifikante Änderung intrakortikaler Fazilitierung. Eine mögliche Erklärung hierfür liegt in einer diskreten Modulation der NMDARezeptor-Aktivität. Diese wäre übereinstimmend mit tierexperimentellen Befunden und humanexperimentellen Ergebnissen zur tDCS, die zeigen, dass die Schwelle zur Induktion inhibierender Plastizität deutlich niedriger als die Schwelle zur Erzeugung fazilitierender Plastizität liegt (Malenka und Bear 2004, Nitsche und Paulus 2001, Nitsche et al. 2003a). Daher scheint es möglich, dass durch kathodale tDCS hervorgerufene Intra-Effekte bereits eine Art von Synapsenmodifikation beinhalten, anodale Intra-tDCS-Effekte jedoch nicht. 


\subsection{Fehlende Wirkung von tDCS auf die I-wave-Aktivität}

Die Ergebnisse der Intra-Strom-Protokolle zeigen keinen Einfluss von tDCS auf die I-WaveFazilitierungskondition.

Das Ausbleiben von Effekten auf die I-wave-Fazilitierung durch Applikation von tDCS stimmt mit der Annahme überein, dass die durch tDCS erzeugten Erregungsänderungen für die Intra-Strombedingungen primär auf Veränderungen der Membranpolarisation beruhen.

I-Wave-Fazilitierung hingegen liegt eine Modifikation von GABAergen und, zu einem kleineren Anteil, auch von glutamatergen Mechanismen zugrunde (Ziemann et al. 1998c). 


\section{Allgemeine Bemerkungen}

Die vorliegende Studie bestätigt die Vermutung, dass die durch kurze, keine Nacheffekte auslösende tDCS hervorgerufenen Modifikationen der kortikospinalen Erregbarkeit weniger von einer Modulation der Synapsen abhängen, als vielmehr von Modifikationen der Membranpolarisation.

Deutlich wird dies in einer durch anodale tDCS induzierten Tendenz zur Vergrößerung der MEPs bei der IO-Kurven-Bestimmung und einer Reduktion derselben bei kathodaler Stimulation. Die I-O-Kurve wird wesentlich durch die Membranpolarisation, nicht aber durch synaptische Mechanismen determiniert. Demgegenüber zeigen die Protokolle für intrakortikale Fazilitierung und Inhibition, sowie für I-wave-Fazilitierung, die durch NMDA- und GABAerge Mechanismen determiniert werden, mit Ausnahme einer Abnahme der intrakortikalen Fazilitierung während kathodaler tDCS, die möglicherweise schon durch diskrete neuroplastische Prozesse bedingt ist, keine nennenswerten Modulationen durch Applikation von tDCS.

Unterstützt wird die Annahme dieses Funktionsmechanismus der tDCS durch einige pharmakologische Studien. Nitsche und Mitarbeiter zeigten in einer Studie mittels der Ionenkanalblocker Carbamazepin (Natriumkanalblocker) und Flunarizin (Kalziumkanalblocker) eine Beteiligung der Ionenkanäle für die Intra-tDCS-Protokolle und bestätigten die Veränderung der Membranpolarisation als Hauptwirkmechanismus (Nitsche et al. 2003c, 2004a). Dahingegen erbrachte die Einnahme von Lorazepam, einem GABAergen Agonisten, und Dextromethorphan, einem NMDA-Rezeptor-Antagonisten, keine Effekte auf Intra-tDCS-Protokolle, während die Einnahme von Dextrometorphan die post-Stimulationseffekte längerer andauernder, neuroplastische Veränderungen erzeugender tDCS für beide Polaritätsbedingungen unterdrückte und somit Hinweise auf eine Beteiligung von NMDA-Rezeptoren an tDCSinduzierter Neuroplastizität liefert (Nitsche et al. 2003, 2004a). NMDA- und GABARezeptoren gelten als hauptsächlich involviert in neuroplastische Veränderungen und in Modulationen auf kortikaler Ebene (Bütefisch et al. 2000, Stefan et al. 2002, Tegenthoff et al.1999). Die vorliegende Untersuchung legt also nahe, dass die durch kurze, keine Nacheffekte hervorrufende, tDCS- induzierten Intra-Effekte auf einer Veränderung des Ruhemembranpotentials beruhen. 
Weitere Studien zeigten, das tDCS in Langzeitversuchen sehr wohl in der Lage ist, kortikale Erregbarkeit längerfristig zu modifizieren. Dazu wurde bei ansonsten vergleichbaren Versuchsprotokollen eine längere Stromstimulationsdauer gewählt; fünf- und siebenminütige tDCS führten sowohl bei kathodaler, als auch bei anodaler Polarisation zu jeweils wenige Minuten anhaltenden Erregbarkeitsveränderungen. Eine Stimulationsdauer von 13 Minuten (anodal) und 9 Minuten (kathodal) erzeugte sogar Modulationen kortikaler Erregbarkeit, die über eine Stunde nach Stimulationsende messbar waren (Nitsche und Paulus 2001, Nitsche et al. 2003b). Eine fMRI-Untersuchung zeigte für selbigen Versuchsaufbau neben den Erregbarkeitsveränderungen gleichgerichtete Aktivitätsänderungen des motorischen Kortex (Baudewig et al. 2001).

Wie bereits beschrieben, konnte gezeigt werden, dass diese neuroplastischen Effekte NMDARezeptor-abhängig sind. Interessanterweise führt die Blockade spannungsabhängiger Ionenkanalrezeptoren allerdings auch zu einer Elimination der Nacheffekte anodaler tDCS, was belegt, das die initiale Veränderung des Ruhemembranpotentials eine notwendige Vorbedingung für die Erzeugung NMDA-abhängiger Neuroplastizität darstellt (Nitsche et al. 2003c).

Trotzdem bietet gerade die Beteiligung der Ionenkanäle an den Intra-tDCS-Effekten interessante Aspekte in Richtung Neuroplastizität. So könnte der durch tDCS induzierte Kalziumeinstrom als Grundvoraussetzung für neuronale Plastizität in kognitiven Studien unterstützend auf lernabhängige Plastizität wirken und diese somit optimieren, ohne sie selbst zu induzieren. Neuere Untersuchungen unterstützen diese These. Nitsche et al. (2003c), sowie Kuo et al. (2008) konnten für eine motorische Lernaufgabe zeigen, dass tDCS während des Lernens die Leistung verbessert, es jedoch unter Applikation von tDCS vor dem Lernen nicht zu einer Verbesserung der Lernleistung kommt. Dieses spricht für eine Beteiligung des intrazellulären Kalziumeinstroms an der Optimierung der Lernleistung, der nur während der tDCS durch die polarisierenden Effekte der tDCS vorhanden ist. Die Stärkung von synaptischen Verbindungen allein, die für die Nacheffekte der tDCS verantwortlich ist, führt dagegen zu keiner verbesserten Lernleistung.

Es ist also, wie oben dargestellt, zu vermuten, dass durch Veränderungen der NMDA- 
Rezeptoreffizienz entstandenen Langzeiteffekten durch tDCS eine Veränderung des Ruhemembranpotentials vorausgeht. Akute Effekte der tDCS induzieren somit Plastizität nicht direkt, erklären aber die Grundlagen zur Entstehung neuroplastischer Veränderungen.

Durch die Ergebnisse dieser Studie erhalten wir nun einen Einblick in die Bahnung und die Entstehung lang anhaltender neuroplastischer Nacheffekte durch die Applikation von tDCS. Diese erfolgt über eine tDCS- induzierte Veränderung des Ruhemembranpotentials, welche auch im Hinblick auf langanhaltende Effekte von tDCS und in Bezug auf Lernvorgänge als Grundvoraussetzung angenommen wird. 


\section{Zusammenfassung}

Kortikale Erregbarkeitsveränderungen sind als neurophysiologische Basis kognitiver Prozesse von großem wissenschaftlichem Interesse. In dieser Arbeit überprüften wir die Wirkungsmechanismen der transkraniellen Stromstimulation mit schwachem Gleichstrom (tDCS), was als nicht invasives Verfahren zerebrale Erregbarkeitsveränderungen beim Menschen induzieren kann. Die Frage, welche kortikalen und intrakortikalen neuronalen Systeme durch kurzzeitig applizierte Stromstimulation moduliert werden, stellt die Grundlange dieser Untersuchung dar.

Dazu wurde bei jungen, gesunden Probanden der Kortex mit kurzer tDCS, welche keine anhaltenden Effekte auf die zerebrale Erregbarkeit nach Beendigung der Stimulation auslöst, stimuliert und mittels transkranieller Magnetstimulation (TMS) kortikale Erregbarkeitsveränderungen über dem Stimulationsareal, der kortikalen Repräsentation eines kleinen Handmuskels, evaluiert. Verschiedene TMS-Techniken sollten hierbei Aufschluss über die Beeinflussbarkeit der kortikalen Erregbarkeit sowie intrakortikaler Fazilitierung und Inhibition geben. Zur Erfassung der Exzitabilität des kortikospinalen Kerngebietes der Muskelrepräsentation wurde bei entspanntem Muskel die Ruheschwelle und bei aktiviertem Muskel die Aktive Schwelle bestimmt. Als Index der kortikospinalen Aktivität größerer Neuronenpopulationen wurde die Input-Output-Kurve erhoben. Intrakortikale Exzitabilität wurde durch die Protokolle der intrakortikalen Inhibition und Fazilitierung, sowie durch die I-wave-Fazilitierung untersucht. Alle verwendeten Protokolle wurden mit anodaler und kathodaler tDCS, sowie ohne Stromstimulation durchgeführt.

Für die Bestimmung der Motorischen Schwellen gab es keine signifikante Differenz zwischen den tDCS-Bedingungen. Dagegen zeigt sich eine signifikante Reduktion unter kathodaler tDCS, sowie eine Tendenz in Richtung Vergrößerung der durch TMS erhobenen Muskelsummenaktionspotentiale (MSAP) unter anodaler tDCS bei der Untersuchung der InputOutput-Kurve. Intrakortikale Inhibition und Fazilitierung zeigen signifikante Veränderungen durch Reduktion der Fazilitierung bei kathodaler tDCS gegenüber der Kontrollbedingung ohne tDCS. Die Darstellung des I-wave-Protokolls zeigt keine Veränderung der I-wave- 
Fazilitierung durch Applikation von tDCS.

Die Untersuchung der Input-Output-Kurve belegt eine, von der tDCS-Polarität abhängige, Modulation der kortikospinaler Erregbarkeit.

Der Einfluss kathodaler tDCS auf die intrakortikale Inhibition und Fazilitierung ist vermutlich durch eine moderate Modulation der NMDA-Rezeptor-Aktivität begründet, während das Fehlen eines Effektes durch anodale tDCS eine erhöhte Schwelle für erregbarkeitssteigernde Exzitabilitätsveränderungen vermuten lässt. Das Ausbleiben von Effekten auf die I-waveAktivität bestätigt den geringen bzw. nicht vorhandenen Effekt von tDCS auf das GABAerge System. 


\section{Literaturverzeichnis}

Abruzzese G. und Trompetto C. (2002): Clinical and research methods for evaluating cortical excitability. J Clin Neurophysiol 19, 307-321.

Antal A., Kinsces T.Z., Nitsche M.A., Bartfai O. und Paulus W. (2004): Excitability changes induced in the primary visual cortex by transcranial direct current stimulation: direct electrophysiological evidence. Invest Ophthalmol Vis Sci 45, 702-707.

Barker A.T., Jalinous R., Freeston I.L. (1985): Non-invasive magnetic stimulation of human motor cortex. Lancet $\underline{1}, 1106-1107$

Baudewig A.T., Nitsche M.A., Fahm J. und Paulus W. (2001): Regional modulation of BOLD MRI responses to human sensorimotor activation by transcranial direct current stimulation. Magn Reson Med 4ㄷ, 196-201.

Bindmann L.J., Lippold O.C. und Redfearn J.W. (1964) The action of brief polarizing currents on the cerebral cortex of the rat (1) during current flow and (2) in the production of long-lasting after-effects. J Physiol 172, 369-382.

Bliss T.V. und Luomo T. (1973): Long lasting potentiation of synaptic transmission in the dentate area of the anesthetized rabbit following stimulation of the perforant path. J Physiol 232, 331-356.

Bütefisch C.M., Davis B.C., Wise S.P., Sawaki L., Kopylev L., Classen J. und Cohen L.G. (2000): Mechanisms of use-dependent plasticity in the human motor cortex. Proc Natl Acad Sci USA $\underline{28}, 3661-3665$.

Buonomano D.V. und Merzenich M.M. (1998): Cortical plasticity: from synapses to maps. Annu Rev Neurosci 21, 149-186.

Castro-Almancos M.A. und Calcagnotto M.E. (1999): Presynaptic long-term potentiation in corticothalamic synapses. J Neurosci 19, 9090-9097.

Charlton C.S., Ridding M.C., Thompson P.D. und Miles T.S. (2003): Prolonged peripheral nerve stimulation induces persistent changes in excitability of human motor cortex. $\mathbf{J}$ Neurol Sci 208, 79-85, 1117-1123 
Chen R. (2000): Studies of human motor physiology with transcranial magnetic stimulation. Muscle Nervesupplement $\underline{9}$, S26-S32.

Chen R. (2004). Interaction between excitatory and inhibitory circuits in the human motor cortex. Exp Brain Res 154, 1-10.

Creutzfeld O.D., Fromm G.H. und Kapp H. (1962): Influence of transcortical d-c currents on cortical neuronal activity. Exp Neurol $\underline{5}, 436-452$

Eysel T.U., Eyding D. und Schweigart G. (1998): Repetetive optical stimulation elicites fast receptive field changes in mature visual cortex. Neuroreport $\underline{5}$, 949-954.

Ferrier D. (1875): Experiments on the brain of monkeys. Proc R Soc Lond 23, 409-430

Fregni F., Boggio P.S., Mansur C.G., Wagner T., Ferreira M.J., Lima M.C., Rigonatti S.P., Marcolin M.A., Freedman S.D., Nitsche M.A. und Pascual-Leone A (2005): Transcranial direct current stimulation of the unaffected hemisphere in stroke patients. Neuroreport $\underline{16}$, 1551-1555.

Fritsch G. und Hitzig E. (1870): Über die elektrische Erregbarkeit des Großhirns. Archiv Anat Physiol Wiss Med 37, 300-332.

Gartside I.B. (1968a): Mechanisms of sustained increases of firing rate of neurons in the rat cerebral cortex after polarization: Role of protein synthesis. Nature $\underline{220}$, 383-384

Gartside I.B. (1968b): Mechanisms of sustained increases of firing rate of neurons in the rat cerebral cortex after polarization: reverberating circuits or modification of synaptic conductance? Nature $\underline{220}, 382-383$

Grafton S.T., Hazeltine E. und Ivry R. (1995): Functional anatomy of sequence learning in normal humans. J Cog Neurosci $\underline{7}, 497-510$.

Grafton S.T., Hazeltine E. und Ivry R. (1998): Abstract and effector-specific representations of motor sequences identified with PET. J Neurosci $\underline{18}$, 9420-9428.

Hess C.W. und Ludin H.P. (1988): Die transkranielle Kortexstimulation mit Magnetfeldpulsen: Methodische und physiologische Grundlagen. Z. EEG-EMG 19, 209-215. 
Hess G. und Donoghue J.P. (1994): Lomg-term potentiation of horizontal connections provides a mechanism to reorganize cortical motor maps. J Neurophysiol 71, 2543-2547.

Huang Y.Z., Edwards M.J., Rounis E., Bhatia K.P. und Rothwell J.C. (2005): Theta burst stimulation of the human motor cortex. Neuron $\underline{45}, 201-206$.

Hummel F., Celnik P., Giraux P., Floel A., Wu W.H., Gerlhoff C. und Cohen L.G. (2005): Effects of non-invasive cortical stimulation on skilled motor function in chronic stroke. Brain $\underline{128}, 490-499$

Kamen G, Caldwell G.E. (1996): Physiology and Interpretation of the electromyogramm. J Clin Neurophysiol $\underline{13}$, 366-384.

Kiers L, Cros D, Chiappa K.H., Fang J (1993): Variability of motor potentials evoked by transcranial magnetic stimulation. Electroencephalgr Clin Neurophysiol $\underline{6}, 415-20$.

Krug M., Lossner B. und Ott T. (1984): Anisomycin blockst he late phase of long-term potentiation in the dentate gyrus of freely moving rats. Brain Res Bull $\underline{13}, 39-42$.

Kujirai T., Caramia M.D., Rothwell J.C., Day B.L., Thompson P.D., Ferbert A., Wroe S., Asselmann P. und Marsden CD (1993): Corticocortical inhibition in human motor cortex. J Physiol 471, 501-519.

Kuo M.-F., Unger M., Liebetanz D., Lang N., Tergau F., Paulus W, Nitsche M.A. (2008): Limited Impactof homeostatic plasticity on motor learning in humans. Neuropsychologica $\underline{46}, 2122-2128$.

Lang N., Nitsche M.A., Paulus W., Rothwell J.C. und Lemon R. (2004): Effects of transcranial DC stimulation over the human motor cortex on corticospinal and transcallosal excitability. Exp Brain Res 156, 439-443.

Leyton A.S.F. und Sherrington C.S. (1917): Observations on the exitable cortex: Of the chimpanzee, orang-utan and gorilla. QJ Exp Physiol 11, 134-222

Liebetanz D., Nitsche M.A., Tergau F. und Paulus W. (2002): Pharmacological approach to synaptic and membrane of the DC-induced neuroplasticity in man. Brain $\underline{125}$, 2238-2247. 
Liepert J., Schwenkreis P., Tegenthoff M. und Malin J.P. (1997): The glutamate antagonist riluzole suppresses intracortical facilitation. J Neural Transm 104, 1207-1214.

Lippold O.C.J. und Redfearn J.W.T. (1964): Mental changes resulting from the passage of small direct currents through the human brain. Brit J Pyschiatry $\underline{110}, 768-772$

Malenka R.C. und Bear M.F. (2004): LTP and LTD: An embarrassment of the riches. Neuron $\underline{44}, 5-21$.

Merton H.B. und Morton P.A. (1980): Stimulation of the cerebral cortex in the intact human subject. Nature $\underline{285}, 227$.

Mills K.R., Boniface S.J., Schubert M (1992): Magnetic brain stimulation with a double coil: the importance of coil orientation. Electroencephalogr Clin Neurophysiol $\underline{85}, 17-21$.

Nitsche M.A. und Paulus W. (2000): Excitability changes induced in human motor cortex by weak direct current stimulation. J Physiol 527, 633-639

Nitsche M.A., Paulus W. (2001): Sustained excitability elevations induced by transcranial DC motor cortex stimulation in humans. Neurology $\underline{57}, 1899-1901$

Nitsche M.A., Liebetanz D., Antal A., Lang N., Tergau F. und Paulus W.: Modulation of cortical excitability by weak direct current stimulation- technical, safety and functional aspects. In: Paulus W., Tergau F., Nitsche M.A., Rothwell J.C., Ziemann U. und Hallett M. (Hrsg.). Transcranial magnetic and transcranial direct current stimulation. Clin Neurophysiol Suppl., Vol.56, Elsevier, Amsterdam, 2003a, 255-276

Nitsche M.A., Nitsche M.S., Klein C.C., Tergau F. und Rothwell J.C. (2003b): Level of action of cathodal DC polarisation induced inhibition of the human motor cortex. Clin Neurophysiol $\underline{114}, 600-604$

Nitsche M.A., Fricke K., Henschke U., Schlitterlau A., Liebetanz D., Lang N., Henning S., Tergau F. und Paulus W. (2003c): Pharmacological modulation of cortical excitability shifts induced by transcranial DC stimulation. J Physiol 553, 293-301.

Nitsche M.A., Schauenburg A., Lang N., Liebetanz D., Exner C., Paulus W., Tergau F. (2003d): 
Facilitation of implicitit motor learning by weak direct current stimulation of the primary motor cortex in the human. J Cog Neurosci 15, 619-626.

Nitsche M.A., Liebetanz D., Schlitterlau A., Henschke U., Fricke K., Lang N., Henning S., Frommann K., Paulus W. und Tergau F. (2004a): GABAergic modulation of DCStimulation-induced motor cortex excitability shifts in human. Eur J Neurosci $\underline{19}, 2720$ 2726.

Nitsche M.A., Jaussi W., Liebetanz D., Lang N., Tergau F. und Paulus W. (2004b): Consolidation of externally induced human motor cortical neuroplasticity by d-cycloserine. Neuropsychopharmacology $\underline{29}, 1573-1578$.

Nitsche M.A., Grundey J., Liebetanz D., Lang N., Tergau F. und Paulus W. (2004c): Catecholaminergic cosolidation of motor cortex plasticity in humans. Cereb Cortex $\underline{14}, 1240-$ 1245.

Nitsche M.A., Seeber A., Frommann K., Klein C.C., Rochford C., Nitsche M.S., Fricke K., Liebetanz D., Lang N., Antal A., Paulus W. und Tergau F. (2005): Modulation parameters of excitability during and after transcranial direct current stimulation of the human motor cortex. J Physiol 568, 291-303.

Pascual-Leone A., Grafman J. und Hallett M. (1994): Modulation of cortical motor outputs maps during development of implicit and explicit knowledge. Science 263, 1287-1289

Purpura D.P. und McMurtry J.G. (1965): Intracellular activities and evoked potential changes during polarization of motor cortex. J Neurophysiol $\underline{28}, 166-185$

Rioult-Pedotti M.S., Friedmann D., Hess G. und Donoghue J.P. (2000): Learning-induced LTP in Neocortex. Science 290, 533-536

Rush S. und Driscoll D.A. (1968): Current distribution in the brain from surface electrodes. Aenest Analg Curr Res 47, 717-723.

Siebner H.R., Lang N., Rizzo V., Nitsche M.A., Paulus W., Lemon R.N. und Rothwell J.C. (2004): Pre-conditioning of low-frequency repetetive transcranial magnetic stimulation with transcranial DC stimulation: evidence for homeostatic plasticity in human motor cortex. J Neurosci 24, 3379-3385. 
Stefan K., Kuensch E., Benecke R., Cohen L.G. und Classen J. (2002): Mechanisms of enhancement of human motor cortex excitability induced by interventional paired associative stimulation. J Physiol 543, 699-708

Tegenthoff M., Witscher K., Schwenkreis P. und Liepert J. (1999): Pharmacological modulation of training-induced plastic changes in human motor cortex. Elektroencephalogr Clin Neurophysiol Suppl 토, 188-196

Terzuolo C.A. und Bullock T.H. (1956): Measurement of imposed voltage gradient adequate to modulate neuronal firing. Proc Nat Acad Sci USA $\underline{42}$, 687-694.

Wassermann E.M. (1998): Risk and safety of repetitive transcranial magnetic stimulation: report and suggest guidelines from International Workshop on the Safety of the Safety of Repetetive Transcranial Stimulation, June 5-7, 1996. Electroencephalogr Clin Neurophysiol $\underline{108}, 1-16$

Ziemann U., Lonnecker S., Steinhoff B.J. und Paulus W. (1996): Effects of antiepileptic drugs on motor cortex excitability in humans: a transcranial magnetic stimulation study. Ann Neurol $\underline{40}, 367-378$.

Ziemann U., Chen R., Cohen L.G. und Hallett M. (1998a): Dextrometorphane decreases the excitability of the human motor cortex. Neurology $\underline{51}, 1320-1324$.

Ziemann U., Tergau F., Wassermann E.M., Wischer S., Hildebrandt J. und Paulus W. (1998b): Demonstration of facilitatory I-wave interaction in the human motor cortex by paired transcranial magnetic stimulation. J Physiol 511, 181-190.

Ziemann U., Tergau F., Wischer S., Hildebrandt J. und Paulus W. (1998c): Pharmacological control of facilitatory I-wave interaction in the human motor cortex. A paired transcranial magnetic stimulation study. Electroencephalogr Clin Neurophysiol 109, 321-330.

Ziemann U. und Rothwell J.C. (2000): I-waves in motor cortex. J Clin Neurophysiol 17, 397 405. 


\section{Danksagung}

Ich danke herzlich Herrn Prof. Dr. med. W. Paulus für die Bereitstellung des Themas und die Aufnahme in das Graduiertenkolleg „Neuroplasticity: From molecules to systems“.

Besonderer Dank gilt Herrn Prof. Dr. med. Michael Nitsche, der mir in den vergangenen Jahren als geduldiger und tatkräftiger Betreuer zur Seite stand und freundlicherweise meine Arbeit als Doktorvater übernahm.

Nicht unerwähnt lassen möchte ich die Techniker Rolf Schneider (Göttingen) und Peter Asselman (London), sowie alle, die namentlich nicht Erwähnung finden konnten und dennoch zum Gelingen der Arbeit beigetragen haben. 


\section{Lebenslauf}

Geboren am 15. September 1975 in Osterode am Harz, besuchte ich von 1982 bis 1995 die Grundschule, Orientierungsstufe und im Anschluss das Tilmann Riemenschneider Gymnasium in Osterode. Mit der Abiturnote „gut“ begann ich im Wintersemester 1995 mein Studium der Humanmedizin an der Georg-August-Universität in Göttingen. Aus gesundheitlichen Gründen unterbrach ich in der Mitte des Sommersemesters (SS) 1996 das Studium, um auf einen Operationstermin zu warten. Zwischenzeitlich übernahm ich eine FSJ (Freiwilliges Soziales Jahr)-Stelle in einem integrativen Kindergarten. Im Sommersemester 1997 konnte ich mein Studium wieder aufnehmen. Die Ärztliche Vorprüfung legte ich im März 1999 ab, es folgten das Erste Staatsexamen im März 2002 und das Zweite Staatsexamen im März 2004. Ich beendete mein Studium im Mai 2005 mit dem Dritten Staatsexamen, in dem ich die Note „sehr gut“" erhielt.

Meine erste Famulatur leistete ich in der gynäkologischen und geburtshilflichen Abteilung der Dres. Herchenhein und Ahrens im Kreiskrankenhaus Herzberg (jetzt Röhnkliniken) ab. Im Sobell Department of Motor Neuroscience and Movement Disorders des National Hospital of Neurology and Neurosurgery in London konnte ich eine weitere Famulatur im Fach meiner Dissertation anschließen. Zuletzt famulierte ich in der Abteilung Innere Medizin bei Prof. Dr. med. Karaus im Ev. Krankenhaus Göttingen-Weende e.V.

Das Praktische Jahr absolvierte ich vollständig im ev. Krankenhaus Göttingen-Weende e.V.. Zunächst in der Abteilung Innere Medizin unter Prof. Dr. med. Karaus, anschließend den chirurgischen Abschnitt im in den Abteilungen Allgemeinchirurgie, Unfallchirurgie und Plastische Chirurgie unter der Leitung der Dres. Fleischer, Dörges und Miericke. Das letzte Tertial arbeitete ich bei Prof. Dr. med. Schenk in der Abteilung für Anästhesiologie und Intensivmedizin.

Neben dem Studium arbeitete ich seit 1998 als studentische Aushilfe auf der internistischen Intensivstation des ev. Krankenhaus Göttingen-Weende e.V..

Im Sommersemester 2000 begann ich mit meiner Forschungsarbeit für die Dissertation in der Abteilung Klinische Neurophysiologie der Universitätsklinik Göttingen unter der Leitung von Prof. Dr. med. Paulus. Zusätzlich nahm ich ab dem Wintersemester 2000 eine Tätigkeit als Hilfswissenschaftlerin in der gleichen Abteilung an. Etwa zum selben Zeitpunkt wurde ich als Kollegiatin in das Graduiertenkolleg „Neuroplasticity: from Molecules to Systems“, initiiert 
von Prof. Dr. med. Paulus, aufgenommen. Im Rahmen einer Rotation mit dem korrespondierenden United College of London (UCL), arbeitete ich von März bis Oktober 2001 im Sobell Department of Motor Neuroscience and Movement Disorders des National Hospital for Neurology and Neurosurgery.

Vom Juni 2005 bis Mai 2010 war ich als Weiterbildungsassistentin der Inneren Abteilung bei Prof. Dr. med. Karaus im Ev. Krankenhaus Göttingen-Weende gGmbH, auch in Rotation mit der Abteilung Geriatrie unter der Leitung von Prof. Dr. med. Nau, angestellt.

Seit Mai 2010 bin als Weiterbildungsassistentin in der Allgemeinarztpraxis von Dr.med. Kessel und Dr.med. Hornig beschäftigt

Meine Kinder kamen im Juni 2002 und im Januar 2007 zur Welt. Das Jahr 2007 verbrachte ich in Elternzeit.

In meiner geringen Freizeit beschäftige ich mich derzeit mit meiner Familie oder mit Sport wie Joggen und Schwimmen.

Sprachlich kann ich mich fließend in Englisch und in Grundkenntnissen in Französisch ausdrücken. Musik und Tanz in klassischer und moderner Form gehören zu meinen derzeit vernachlässigten, jedoch nicht vergessenen Interessen. 